\title{
Interaction of Excitation and Inhibition in Anteroventral Cochlear Nucleus Neurons That Receive Large Endbulb Synaptic Endings
}

\author{
Cornelia Kopp-Scheinpflug, ${ }^{1}$ Susanne Dehmel, ${ }^{1}$ Gerd J. Dörrscheidt, ${ }^{2}$ and Rudolf Rübsamen ${ }^{1}$ \\ ${ }^{1}$ Department of Neurobiology, University of Leipzig, 04103 Leipzig, Germany, and 2Department of General Zoology and \\ Neurobiology, Ruhr-University Bochum, Bochum, 44801 Germany
}

Spherical bushy cells (SBCs) of the anteroventral cochlear nucleus (AVCN) receive their main excitatory input from auditory nerve fibers (ANFs) through large synapses, endbulbs of Held. These cells are also the target of inhibitory inputs whose function is not well understood. The present study examines the role of inhibition in the encoding of low-frequency sounds in the gerbil's AVCN. The presynaptic action potentials of endbulb terminals and postsynaptic action potentials of SBCs were monitored simultaneously in extracellular single-unit recordings in vivo. An input-output analysis of presynaptic and postsynaptic activity was performed for both spontaneous and acoustically driven activity. Two-tone stimulation and neuropharmacological experiments allowed the effects of neuronal inhibition and cochlear suppression on SBC activity to be distinguished.

Ninety-one percent of SBCs showed significant neuronal inhibition. Inhibitory sidebands enclosed the high- or low- frequency, or both, sides of the excitatory areas of these units; this was reflected as a presynaptic to postsynaptic increase in frequency selectivity of up to one octave. Inhibition also affected the level-dependent responses at the characteristic frequency. Although in all units the presynaptic recordings showed monotonic rate-level functions, this was the case in only half of the postsynaptic recordings. In the other half of SBCs, postsynaptic inhibitory areas overlapped the excitatory areas, resulting in nonmonotonic rate-level functions. The results demonstrate that the sound-evoked spike activity of SBCs reflects the integration of acoustically driven excitatory and inhibitory input. The inhibition specifically affects the processing of the spectral, temporal, and intensity cues of acoustic signals.

Key words: prepotential units; endbulb of Held; cochlear suppression; neuronal inhibition; in vivo physiology; bicuculline; strychnine; gerbil; spherical bushy cells; cochlear nucleus
The cochlear nucleus consists of three subdivisions: the anteroventral cochlear nucleus (AVCN), the posteroventral cochlear nucleus, and the dorsal cochlear nucleus, each of which establishes the origins of several monaural and binaural ascending pathways (for review, see Irvine, 1986). It was believed previously that the AVCN serves primarily as a relay transmitting a highfidelity copy of the activity of the auditory nerve to more central auditory brainstem nuclei (Pfeiffer, 1966a,b; Rose et al., 1974). This was demonstrated by morphological data which show that the principal neurons of the AVCN, the spherical bushy cells (SBCs), are innervated by only two to four auditory nerve fibers (ANFs) that form large synaptic endbulbs on the somata of the neurons (Brawer and Morest, 1975; Ryugo and Sento, 1991; Bazwinsky et al., 1999). Because of this specific synaptic configuration, electrophysiological recordings using extracellular electrodes can detect the postsynaptic action potentials of SBCs together with a preceding "prepotential" (PP), indicating the presynaptic afferent ANF input (Pfeiffer, 1966a; Shofner and Young, 1985; Young et al., 1988; Winter and Palmer, 1990; Winter et al., 1990).

Our attempt to study signal transmission at the AVCN endbulb synapses was motivated by more recent anatomical and physiological results which indicate that the AVCN receives not only

Received June 13, 2002; revised Sept. 12, 2002; accepted Oct. 3, 2002.

This work was supported by Deutsche Forschungsgemeinschaft (DFG) Graduiertenkolleg 250/1-96 and DFG Ru 390-15/1 and 15/2. We thank Drs. Donata Oertel and W. Lippe for helpful comments on earlier versions of this manuscript.

Correspondence should be addressed to R. Rübsamen, Department of Neurobiology, University of Leipzig, Talstrasse 33, 04103 Leipzig, Germany. E-mail: rueb@rz.uni-leipzig.de.

Copyright (๑) 2002 Society for Neuroscience $0270-6474 / 02 / 2211004-15 \$ 15.00 / 0$ excitatory ANF input but also inhibitory inputs. The SBCs receive inhibitory projections from the dorsal and ventral cochlear nucleus (Wu and Oertel, 1986; Roberts and Ribak, 1987; Wenthold et al., 1987; Snyder and Leake, 1988; Oertel et al., 1990; Wickesberg and Oertel, 1990; Kolston et al., 1992; Ferragamo et al., 1998) and from higher order auditory brainstem nuclei [medial nucleus of trapezoid body and superior paraolivary nucleus (Schofield, 1991, 1994); lateral and ventral nucleus of trapezoid body (Covey et al., 1984; Warr and Beck, 1996)]. Pharmacological studies in vivo and in vitro confirmed that neurons in the AVCN are sensitive to GABA and glycine (Wu and Oertel, 1986; Walsh et al., 1990; Wickesberg and Oertel, 1990; Caspary et al., 1993, 1994; Ebert and Ostwald, 1995a,b).

In our study we made use of the fact that the incidence and the timing of the presynaptic and the postsynaptic signal components of SBC recordings can be analyzed separately, and the result can be used to evaluate the input-output function at the ANF-SBC synapse. This offers the rare opportunity to study in vivo the presynaptic to postsynaptic signal transmission in the mammalian auditory system using acoustic stimulation. Comparison between presynaptic and postsynaptic activity during two-tone stimulation allows the effects of inhibition on SBCs to be differentiated from those of cochlear suppression (Sachs and Kiang, 1968). Pharmacological experiments enable the effects of GABAergic and glycinergic inhibition to be differentiated from the effects of other cellular mechanisms onto synaptic transmission.

The results gave strong evidence that inhibition alters the frequency tuning and the level-dependent responses and increases the precision of encoding of the signal onset. This suggests that the 
neuronal activity is the result of excitation and inhibition already at the level of second-order neurons of the auditory system.

\section{MATERIALS AND METHODS}

\section{Animals and animal care}

The experiments were performed at the Neurobiology Laboratories of the Zoological Department of the University of Leipzig (Germany). All experimental procedures were approved by the Saxonian District Government, Leipzig. Adult pigmented (agouti) Mongolian gerbils (Meriones unguiculatus), ages 3-6 months and weighing 50-80 gm, were used in the experiments. The animals were obtained from the animal care facilities of the Zoological Department of the University of Leipzig.

\section{Surgical preparation}

During the experiments and the surgical preparation, the animals were anesthetized with an initial dose of $0.3 \mathrm{ml} / 100 \mathrm{gm}$ body weight of a 10:1 mixture of ketamine hydrochloride $(0.13 \mathrm{mg} / \mathrm{g}$ body weight; Parke-Davis, Courbevoie, France) and xylazine hydrochloride $(0.005 \mathrm{mg} / \mathrm{g}$ body weight; Bayer, Wuppertal, Germany). During the recording experiments, a constant level of anesthesia was maintained by hourly injections of one-third of the initial dose. The skull of the experimental animal was exposed along the dorsal midsagittal line, and a small metal bolt for supporting the animal in the stereotaxic recording device was glued to the bone overlaying the forebrain. Two holes were drilled in the skull 2000-2300 $\mu \mathrm{m}$ caudal to the lambda suture, which correspond to positions above the rostral third of the cerebellum. The first drill hole, located $1500 \mu \mathrm{m}$ lateral to the midline, was used to position the reference electrode in the superficial cerebellum. For the insertion of single-barrel electrodes, the second drill hole (500 $\mu \mathrm{m}$ diameter) was located over the midline, and the recording electrode was angled at $28-30^{\circ}$ to the midsagittal plane. For multibarrel electrodes, the drill hole $(1000 \mu \mathrm{m}$ diameter) was located $1000 \mu \mathrm{m}$ parasagittally.

\section{Acoustic stimulation}

All acoustic stimuli were digitally generated with 16 bit accuracy by a $486 / 33$ computer. The stimuli were delivered at $250 \mathrm{k}$ samples per second per channel through a two-channel, 14 bit analog-to-digital (A/D) converter including a custom-made low-pass resynthesis filter $(50 \mathrm{kHz}$ cutoff) and a software-controlled attenuator $(0-120 \mathrm{~dB}$ in $1 \mathrm{~dB}$ steps). A source selector allowed the two output signals to be directed either separately or concurrently to the two channels of a stereo amplifier (Microline) that drove the sound transducers. Using the acoustic couplers of DT48 headphones (Beyer Dynamics), custom-made acoustic transducers were designed for "near-field" stimulation of the ear. Sound was delivered through a plastic tube $(5 \mathrm{~mm}$ outer diameter at the end) placed in the funnel of the outer ear $\sim 5 \mathrm{~mm}$ from the animal's eardrum. The frequency characteristic of the transducer was measured with a $1 / 4$ inch condenser microphone (Bruel and Kjaer type 2619) coupled to a short plastic tube mimicking the conditions in the ear canal. A computercontrolled procedure determined for 50 frequencies per decade the sound pressure levels (SPLs) at a defined input voltage. The data were stored in a computer file that was used during experiments for on-line level correction of stimulus intensities.

\section{Data collection and analysis}

All recording experiments were performed in a sound-attenuated chamber (Type 400, Industrial Acoustics). During the experiments the body temperature was kept between 36 and $37.5^{\circ} \mathrm{C}$ by positioning the animal on a temperature-controlled heating pad and maintaining the temperature of the recording chamber at $25-30^{\circ} \mathrm{C}$.

Multiunit mapping. The tonotopic organization of the ventral cochlear nucleus of the gerbil is comparable with that of other mammals [african mole rat (Müller et al., 1992); gerbil (Müller, 1996; Cant and Hyson, 1992)]. In the first recording session for each animal, the stereotaxic coordinates of the AVCN were determined by on-line analysis of acoustically evoked multiunit activity. For this, glass micropipettes (Clark Electromedical Instruments, Pangbourne, UK) filled with $3 \mathrm{M} \mathrm{KCl}$ and having impedances of 5-10 M $\Omega$ were used. With use of monaural tone-burst stimulation, the characteristic frequencies $(\mathrm{CFs})$ of multiunits were measured every $100 \mu \mathrm{m}$ in several penetrations in the estimated position of the AVCN (test range, $0.1-50 \mathrm{kHz}$ and $0-90 \mathrm{~dB}$ SPL). The cochlear nucleus, located at a dorsolateral position in the rostral medulla oblongata, was reached at a penetration depth of 5900-6500 $\mu \mathrm{m}$, de- pending on the size of the animal. The distribution of the CFs along the rostrocaudal, dorsoventral, and mediolateral dimensions of the acoustically excitable area enabled the demarcation of the anterior division of the AVCN on physiological criteria. In brief, in the AVCN high frequencies are located caudally and dorsally, and low frequencies are located rostrally and ventrally. Furthermore, electrode penetrations that hit the posteroventral cochlear nucleus first have to pass through the dorsal cochlear nucleus. Because the latter subnucleus also has a dorsoventral high- to low-frequency axis, there was always a recognizable discontinuity in CF values when the electrode moved out of the dorsal and into the posteroventral cochlear nucleus.

Single-unit recordings. After multiunit mapping, recordings from single units were made through higher impedance glass micropipettes. Best signal-to-noise ratios and the highest probability of being able to simultaneously record PPs and action potentials were obtained with electrode impedances of $15-30 \mathrm{M} \Omega$.

Single-unit recordings with neuropharmacological manipulations were performed with five-barreled piggyback electrodes [tip diameter, $8 \mu \mathrm{m}$; recording barrel protruding 5-10 $\mu \mathrm{m} ; 10-20 \mathrm{M} \Omega$; after Havey and Caspary (1980)]. Drugs [strychnine HCl, 7 mM, pH 3 (RBI-Sigma, Taufkirchen, Germany); bicuculline methiodide, 5 mM, pH 3 (RBISigma)] were applied iontophoretically $(+15-50 \mathrm{nA})$. Holding current for each barrel was $-15 \mathrm{nA}$. A balancing or summating channel was used to alleviate current effects (barrel filled with $1 \mathrm{~m}$ sodium acetate).

The activity of isolated single units was bandpass filtered $(0.3-10 \mathrm{kHz})$ and amplified to the voltage range of the spike discriminator and the A/D converter. Single units were identified by three criteria: (1) relatively constant spike height, (2) constant waveform, and (3) large signalto-noise ratio $(>2)$. For acquisition of single-unit activity, the amplified recording signal was delivered to a custom-made window discriminator followed by an event-timer personal computer interface. During a single recording, the window discriminator was set to be triggered by either the PPs or the postsynaptic action potentials using a combined slope, level, and dead-time criterion (see Fig. $2 A$ ). This allowed the two types of signals to be differentiated for analysis. The discriminated spike times were acquired with $100 \mu \mathrm{sec}$ resolution using custom-written real-time computer programs. Analog waveforms of the signals, as presented in Figures 1 and 2, were recorded with an A/D conversion rate of 20,000/sec (50 $\mu$ sec sample interval).

The excitatory response areas for the action potentials and the PPs were measured separately by random presentation of pure-tone pulses (100 msec duration, $5 \mathrm{msec}$ rise-fall time, $50 \mathrm{msec}$ interstimulus interval) within a given matrix of $16 \times 15$ frequency/intensity pairs $(240$ combinations). Each frequency/intensity combination was presented five times in a predefined frequency/intensity array. The timing and the number of spikes were measured during the $100 \mathrm{msec}$ period of stimulus presentation and in the absence of acoustic stimulation [spontaneous rate (SR)]. From these data the $\mathrm{CF}$, threshold, bandwidth of excitation, and ratelevel functions (at the CF of the units) were calculated.

Analysis of spike recordings. Besides visualizing spike responses [peristimulus time histogram (PSTH), spike counts/rates, dot display], we also computed rate-level functions and frequency-threshold curves. Typical responses of AVCN neurons are shown in Figure 5. In Figure 5, $A$ and $B$, the height of each bar represents the number of spikes (logarithmic scale, maximum 50 spikes) during a single stimulus presentation of the given frequency and intensity combination. The neuronal response area shown in Figure $5 A 2$ was defined by the range of frequency/intensity combinations that evoked discharge rates at or above the $10 \%$ significance level above SR. SR was defined by the discharge rate with maximal attenuation $(120 \mathrm{~dB})$ of the stimulus (bottom-most row in the graphs). The outline of the response area defined the frequency-threshold curve of the unit, from which the $\mathrm{CF}$ and the $\mathrm{CF}$ threshold were determined (see Fig. 5A2). Each successive contour line represents an increase in firing rate equivalent to the upper boundary of the $10 \%$ significance interval of the discharge rate shown by the contour line just below. That is, the successive contour lines represent increasing discharge rates in steps defined by successive $10 \%$ significance intervals.

Statistical analyses of the data were performed with SigmaStat/SigmaPlot (SPSS Science, Chicago, IL). For normally distributed data, results are expressed as means $\pm \mathrm{SD}$, and statistical significance was assessed using the Student's $t$ test or paired $t$ test. For other distributions, results are expressed as medians ( 25 and $75 \%$ quartiles), and significance was assessed using the Wilcoxon signed rank test or the Mann-Whitney rank sum test. 
Waveform analysis. The number of independent waveforms present in recordings of AVCN activity was determined by applying the principal component (PC) analysis, followed by a cluster analysis. This analysis was first introduced into neurophysiology by Abeles and Goldstein (1977) and has since found various applications in this field (Wiener and Richmond, 1999). Principal component analysis showed that waveform recordings of AVCN activity were separable into two distinctive components, i.e., complex waveforms (spike-preceding PPs plus postsynaptic spikes) and isolated PPs. In our application, all waveforms ( $2.5 \mathrm{msec}$ time epochs) that met a user-defined trigger criterion comprising upper and lower thresholds, slope direction, and dead-time were selected from digitized waveform recordings. Principal component analysis is able to describe each waveform from this ensemble as the weighted sum of a number of basic waveforms, the PCs. Depending on the variance of the ensemble, only a few PCs and a correspondingly small number of weights may give a sufficient description of the great majority of the original waveforms. Goodness of fit is expressed as the quotient of "variance of the approximating set of waveforms"/"variance of the original ensemble of waveforms," so that $100 \%$ indicates perfect approximation. Preceding the analysis, the selected signals were multiplied by a windowing function to enhance the weight of the center region (Rabiner and Gold, 1975). The basic functions and weights were then determined following the Karhunen-Loeve expansion as described by Glaser and Ruchkin (1976). Figure 2 shows the results from one representative unit.

Verification of recording sites. In each animal, all recording sites were verified histologically using horseradish peroxidase (HRP) marking. At the end of the recording session, the recording electrode was replaced with a glass micropipette filled with a solution of $9 \% \mathrm{HRP}$ in $0.9 \% \mathrm{NaCl}$, which was injected iontophoretically ( $2 \mu \mathrm{A}$ for $5 \mathrm{~min}$ ) into the recording site. The position of the HRP electrode was confirmed by measuring the $\mathrm{CF}$, threshold, and PSTH of the neuronal activity at the injection site. Alternatively, HRP was injected directly through one of the barrels of the multibarrel electrodes. The animal was allowed to survive for $24 \mathrm{hr}$ and was then perfused via the left ventricle of the heart with $0.9 \% \mathrm{NaCl}$ solution followed by fixative $(2.5 \%$ paraformaldehyde in $0.1 \mathrm{M}$ phosphate buffer, $\mathrm{pH}$ 7.4) for 20-25 min. The brain was cut on a vibratome, and the tissue sections $(100 \mu \mathrm{m})$ were reacted using the $3,3^{\prime}$-diaminobenzidine reaction to visualize the HRP mark (Adams 1981). After staining with cresyl violet, the tissue sections were examined under the light microscope, and the electrode tracks and recording sites were reconstructed.

\section{RESULTS}

Sound-evoked activity was recorded in the rostral-most $800 \mu \mathrm{m}$ of the nucleus, which has a rostral-to-caudal extension of $\sim 2.5 \mathrm{~mm}$ (Rübsamen et al., 1994). This was done because the rostral AVCN includes a nearly homogenous population of neurons, the SBCs (Irvine, 1986; Cant and Hyson, 1992; Ostapoff et al., 1994; Bazwinsky et al., 1999), which in this part represent frequencies below 3-6 kHz and show the largest endbulb terminals (Rouiller et al., 1986). Therefore a higher percentage of PP recordings could be expected. We recorded 194 units of 31 gerbils. Of this sample, 85 units were studied using iontophoretic application of bicuculline or strychnine. Because a comprehensive acquisition of the pharmacological effects required stable recording conditions for a minimum of $30 \mathrm{~min}$, it could be performed on only 19 units, from which 15 units were tested for the effect of bicuculline and 4 units were tested for the effect of strychnine. The pharmacological data presented below show the results of those units (5 of 15 bicuculline; 4 of 4 strychnine) that responded with a significant overall increase in discharge rate after block of inhibition. All recording sites were verified by HRP injections (see Materials and Methods).

\section{Waveform of neuronal discharges}

The discharges of $68 \%$ (74 of 109) of the neurons recorded in the present study with single-barrel electrodes showed complex waveforms that were comparable with those in the cat AVCN (Pfeiffer, 1966a). These waveforms consisted of bipolar action potentials preceded immediately by PPs (Fig. $1 A-C$ ); the corresponding

\section{A $075-1902$}
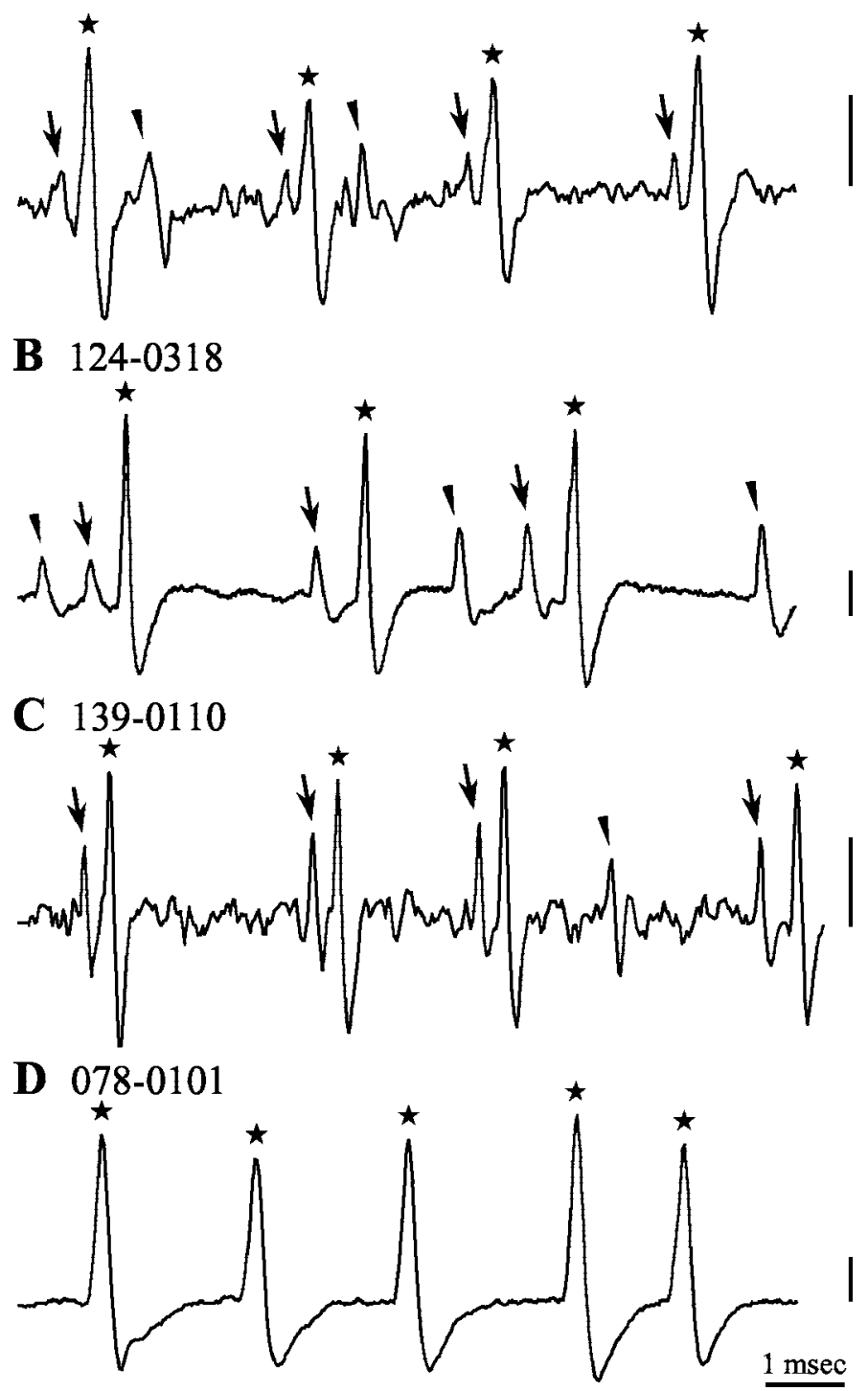

Figure 1. Extracellularly recorded tone-evoked neuronal discharges of four AVCN units. $A-C$, Units with PPs [CF: $1.2 \mathrm{kHz}(A) ; 0.8 \mathrm{kHz}(B) ; 1.5$ $\mathrm{kHz}(C)$ ]. Postsynaptic action potentials are marked by asterisks, PPs preceding each action potential are marked by arrows, and isolated PPs are marked by arrowheads. D, Unit lacking PPs (CF: $2.1 \mathrm{kHz})$. Vertical bars indicate $0.5 \mathrm{mV}$.

units are referred to as "PP units." Forty-two of these PP units provided the quantitative data for the findings described below. The PPs preceded the action potentials by $0.83 \pm 0.04 \mathrm{msec}$ (measured peak presynaptic to peak postsynaptic potential; $n=$ 315 signals in five units). In between PP units and intermixed with them, other units were recorded that either lacked PPs (Fig. 1D) or had PPs that were too small in amplitude to be reliably distinguished from the noise floor (35 of $109=32 \%$ of the entire ensemble). Because these recordings also showed primary-like PSTHs, they probably reflect SBC activity recorded with an unfavorable spatial relation between the tip of the electrode and the endbulb terminal. Chopper units, which were not numerous in the targeted low-frequency area, never showed signs of PPs. An inspection of the conditions under which PP units were reliably recorded in the AVCN revealed that the amplitude of PPs decreased in units with CFs above 2-3 kHz (Kopp-Scheinpflug, 1999). 
In many recordings from PP units, a "second" type of signal occurred that by shape and size resembled PPs but was not immediately followed by spike discharges (Fig. $1 A-C$ ). These signals will be referred to as "isolated PPs." Pfeiffer (1966a) reported previously in his recordings from the cat AVCN what he termed "spike failures." These were small-sized signals that, as in our recordings, were not followed by action potentials. However, he did not subject these signals to a more detailed analysis. In the present study, the term "presynaptic activity" will be used when referring jointly to the spike-preceding PPs and to the isolated PPs. The term "postsynaptic activity" is used to refer to the action potential discharges of SBCs.

We will argue that the neuronal element generating the isolated PPs is the same presynaptic endbulb that also gives rise to the spike-preceding PPs and that the isolated PPs represent endbulb discharges that have failed to evoke postsynaptic spikes. If this interpretation is correct, the recordings should have no more than two different types of waveforms: the spike-preceding PPs followed by postsynaptic action potentials and the isolated PPs. If, alternatively, the recording had sampled the discharges of multiple units, the analysis should reveal not only two waveforms but, with the same probability, three or more waveforms. Even if a multiunit recording sampled only two independent units, the spikes occurring mixed together with variable timing would superimpose, resulting in more than two waveforms. To determine how many independent waveforms were represented in a recording, principal component analysis was performed on digitized waveform recordings of $21 \mathrm{PP}$ units. The results of this analysis provide strong evidence that only two waveforms are distinguishable in the recordings: the spike-preceding PPs followed by postsynaptic spikes and the isolated PPs (Fig. $2 B-G$ ). The principal component analysis yielded comparable results for all 21 units, as indicated by the $85-95 \%$ efficiency by which the first two principal components jointly describe the recorded signals.

A number of other observations corroborate the conclusion that isolated PPs and spike-preceding PPs originate from the same source, namely the presynaptic endbulb terminal. (1) During recording, isolated PPs and spike-preceding PPs always appeared and disappeared in concert, e.g., as the electrode was moved. (2) Isolated PPs were observed only in recordings from PP units and never in recordings from the 35 of 109 AVCN units that lacked spike-preceding PPs. (3) Spike-preceding PPs and isolated PPs are comparable in size and shape, e.g., both signals have comparable onset slopes and thereby could be selected by the same slope criterion $(0.15 \mathrm{mV} / \mathrm{msec})$.

If, in fact, the spike-preceding PPs and the isolated PPs reflect the activity of the same endbulb, one has the opportunity to simultaneously record and compare the activity of the SBC and its afferent input (spike-preceding PP plus isolated PP). Here, we used this approach to evaluate the presynaptic-to-postsynaptic input-output functions and consider the influence of neuronal inhibition on the activity of SBCs.

\section{Comparison of presynaptic and postsynaptic activity}

\section{Spontaneous and sound-evoked discharge rate}

Presynaptic SR ranged from 2 to 245 spikes per second (sps) (mean, $96 \pm 52$ sps; 42 units). This was significantly larger than the SR measured postsynaptically (1-188 sps; mean, $60 \pm 44$ sps; paired $t$ test; $p \leq 0.001)$. The percentage of isolated PPs represents a "failure rate" $(100 \%=$ spike-preceding PP + isolated PP $)$; values ranged from 4 to $93 \%$ (mean, $42 \%$ ). During the applica- tion of strychnine or bicuculline in 10 of 19 units, the SR was increased from $62 \pm 45$ to $71 \pm 46$ sps.

Sound-evoked discharge rates were measured during pure-tone stimulation at the $\mathrm{CF}$ of each unit, $50 \mathrm{~dB}$ above threshold. Presynaptic rates ranged from 92 to $602 \mathrm{sps}$ (mean, $270 \pm 93 \mathrm{sps} ; n=$ 42). This was significantly larger than the sound-driven rates measured postsynaptically (16-360 sps; mean, $119 \pm 68$ sps; paired $t$ test; $p \leq 0.001$ ). The failure rates ranged from 4 to $94 \%$ (mean, $54 \%$ ). During drug application in 9 of 19 units, the sound-evoked rates at CF $50 \mathrm{~dB}$ above threshold were significantly increased from $136 \pm 84$ to $244 \pm 138 \mathrm{sps}$ (paired $t$ test; $p=0.011$ ).

\section{Response pattern}

In response to tone bursts at $\mathrm{CF}$ and at $50 \mathrm{~dB}$ above threshold, all units showed phasic-tonic PSTHs in their presynaptic activity, and most units also showed them in their postsynaptic activity (Fig. 3). This primary-like PSTH is known to be typical for SBCs (Pfeiffer, 1966b; Shofner and Young, 1985; Young et al., 1988). After the offset of the stimuli, spike rates were reduced below SR for $20-50 \mathrm{msec}$ in both the presynaptic and postsynaptic activity. The duration of this off-suppression correlated with the magnitude of the excitatory response.

The timing of the onset is sharpened postsynaptically in SBCs because of a rate reduction during the remainder of the soundevoked activity (Fig. 3). This is reflected by a reduction of the width of the onset peak (width at $75 \%$ of the peak: presynaptic $8.0 \pm 3.8 \mathrm{msec}$; postsynaptic $4.1 \pm 4 \mathrm{msec}$; paired $t$ test; $p=$ $0.003)$. Additionally, the rate reduction causes an enhancement of the peak relative to the steady-state activity. Pharmacological experiments revealed that the postsynaptic reduction of the steady-state response is caused by inhibition (Fig. 3I-P). As can be seen from the summed PSTHs in Figure $3 G, H, O, P$, the steady-state response ( $30-90 \mathrm{msec}$ ) was reduced by $57 \%$ from 143 sps presynaptically to 62 sps postsynaptically $(n=22)$ and was doubled from 14 sps (PREDRUG) to 28 sps during drug application $(n=7)$. The relative enhancement of the peak was quantified by the "peak-over-total ratio," which was significantly larger postsynaptically than presynaptically ( $n=22$; paired $t$ test; $p \leq$ 0.001 ) (Fig. 4). After the postsynaptic inhibition was blocked, only a tendency for the decrease of the peak-over-total ratios was seen ( $n=7$; Wilcoxon signed rank test; $p=0.08$; NS) (Fig. 4).

In contrast to presynaptic responses, postsynaptic PSTHs could vary within the response area of the unit. Such variations were most prominent in units that had upper thresholds (high sound levels at which the rate fell equal to or below SR) or in units in which the excitatory response areas were bordered by pronounced inhibitory sidebands (see below). In those, the PSTHs varied as a function of stimulus level and stimulus frequency from the phasictonic prototype to a more phasic on or phasic on-off type (Fig. $5 B 5$, inset). Changes were most distinct at high stimulus levels and near the high- and low-frequency borders of the response areas.

\section{Frequency selectivity}

Complete presynaptic and postsynaptic excitatory response areas were acquired for 42 PP units. Postsynaptically, the response areas tended to be more variable in shape and threshold than presynaptic ones. A feature of half the postsynaptic response areas, never seen in presynaptic recordings, was a restriction of the response bandwidth toward the higher stimulus levels. In many of those units the reduction in discharge rate even resulted in the formation of upper thresholds. In most cases the upper thresholds were in the range of 60-90 dB SPL, but they could also 
A

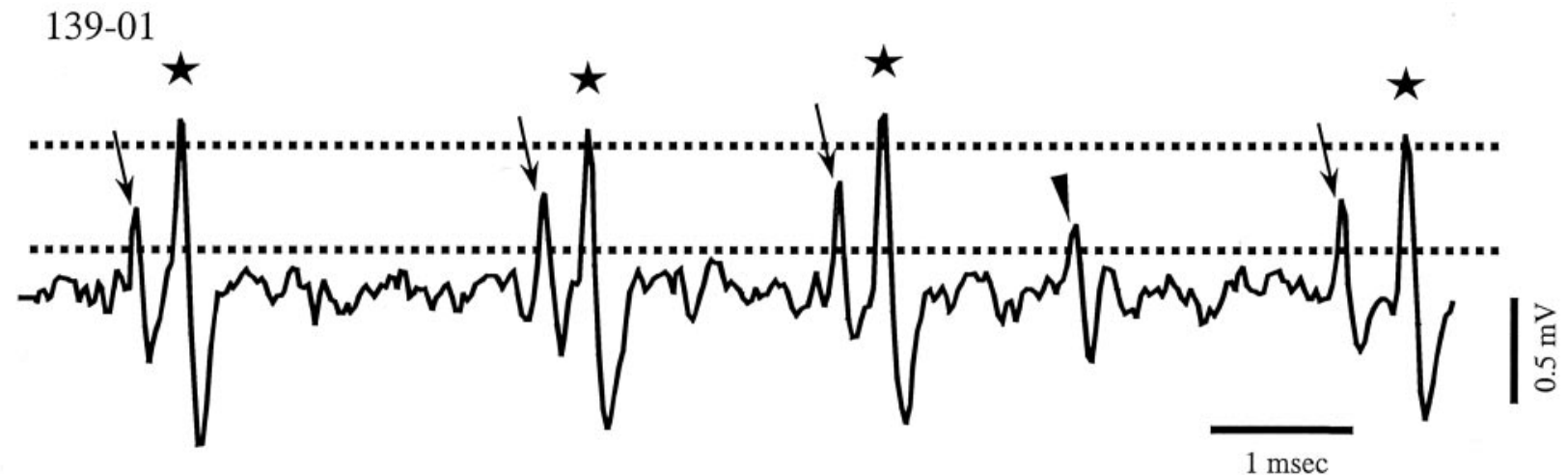

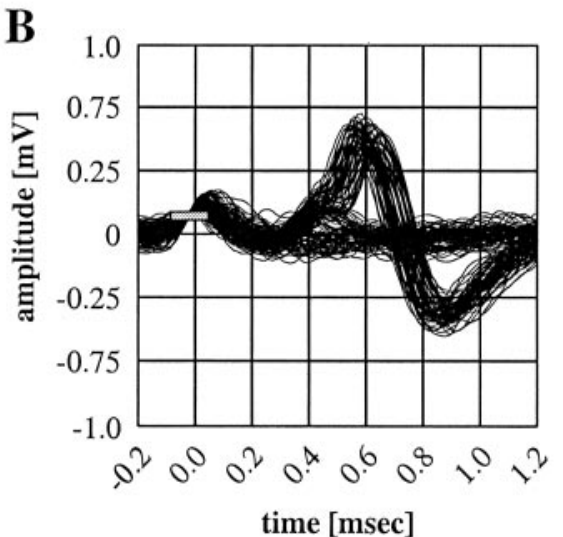

E

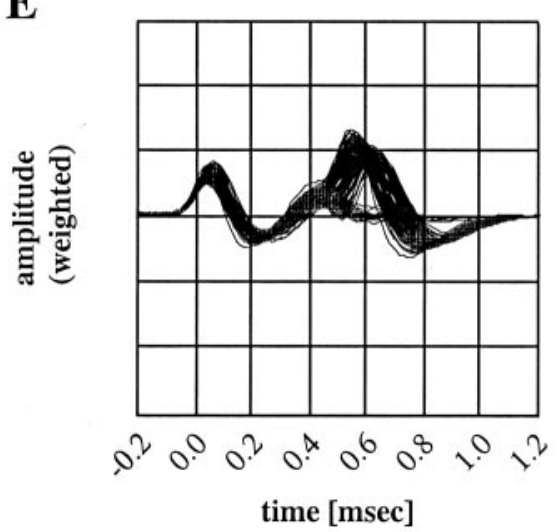

C

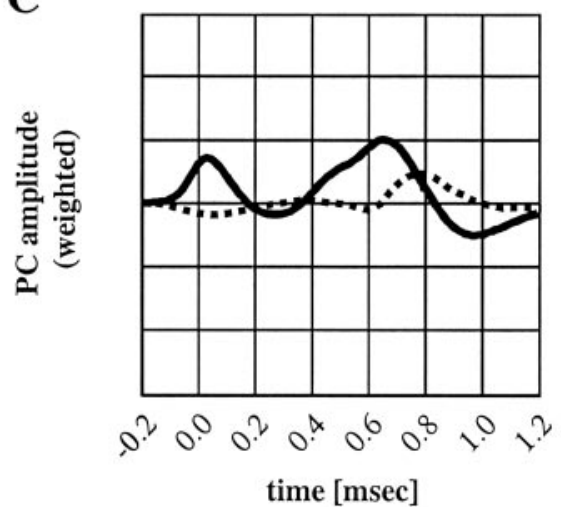

F

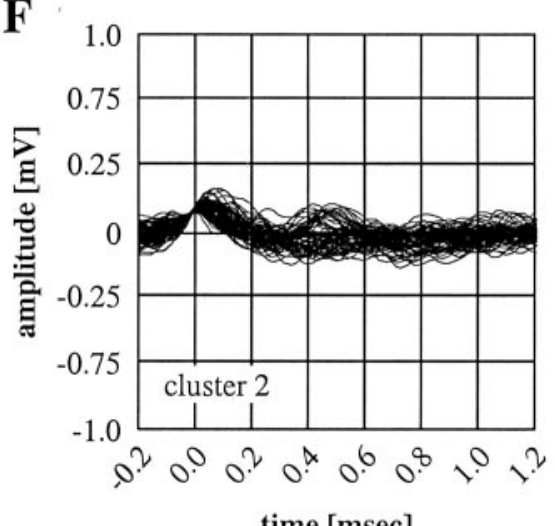

D

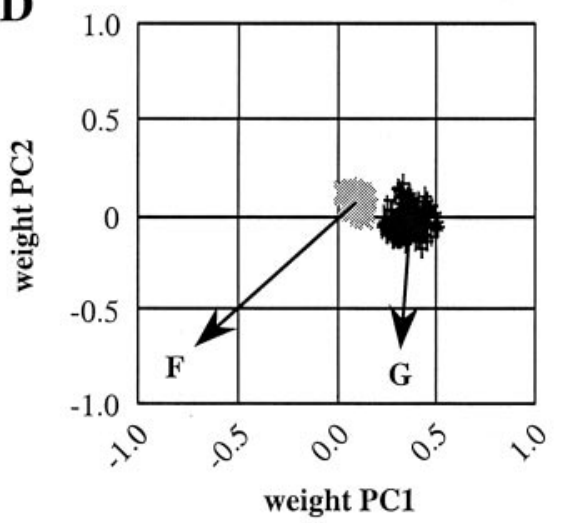

G

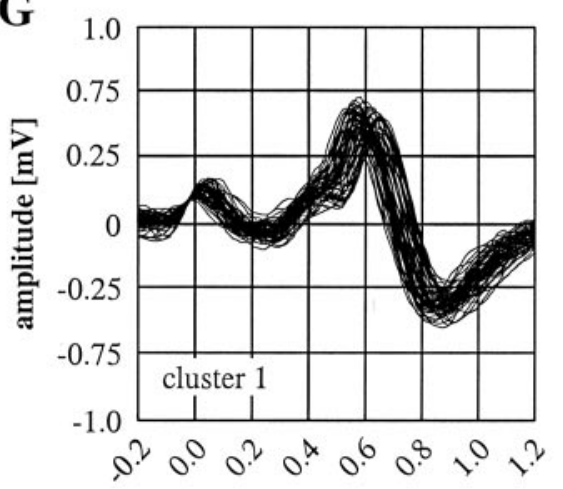

time $[\mathrm{msec}]$

Figure 2. Separation of presynaptic and postsynaptic signal components (unit 139-01). A, Setting of trigger levels for the acquisition of response maps (see below). Presynaptic and postsynaptic discharge activity can be separated by different thresholds (bottom and top dotted lines). Additionally, a preset dead time of $1 \mathrm{msec}$ prevents double triggers of postsynaptic action potentials after PPs in the PP recordings. Asterisks indicate postsynaptic action potentials; arrows and arrowheads indicate PPs. $B-G$, Separation of signal constituents using PC analysis. $B$, Superimposed waveforms of 301 discharges. Trigger level was set to the onset slope of PPs. The gray bar (at $0.12 \mathrm{mV}$ ) indicates the upper threshold of the combined threshold and slope criterion. $C$, The first two PCs (PC1, black line; PC2, dotted line) of the signals shown in $B$ multiplied by their root-mean-square weight. The whole of the signals could be sufficiently described by the first two PCs with an $87 \%$ efficiency; i.e., each of the 301 recorded signals can be adequately explained by the weighted sum of two functions, PC1 and PC2. D, On the basis of PC1 and PC2 weights, the potentials are easily separated by a cluster algorithm into two clusters. In this scatter plot, the cluster with the higher PC1 weights (cluster 1, black; $n=233$ ) represents the complex waveforms, and the cluster with the lower PC1 weights (cluster 2, gray; $n=68$ ) identifies the isolated PPs. E, Resynthesis of all 301 signals from the two PC time courses in $C$ and the individual weight pairs in $D$. The waveforms split up into two subsets, corresponding to either isolated PPs or spike-preceding PPs followed by postsynaptic spikes. $F$ and $G$ show the original waveforms from $B$ related to cluster 1 (spike-preceding PPs followed by postsynaptic spikes) and to cluster 2 (isolated PPs), respectively.

be as low as $40 \mathrm{~dB}$ SPL. Figure 5, $A$ and $B$, depicts two representative units with recordings of their presynaptic activity indicated in the top panels, and recordings of their postsynaptic activity indicated in the bottom panels. Both units showed a presynaptic to postsynaptic reduction in spontaneous and sound-evoked activity in the whole response area (Fig. 5, left column). Also, the number of iso-response contours was reduced, indicating a reduction of the dynamic range (Fig. 5, center column). The rate-level functions turned from a monotonic to a nonmonotonic course (Fig. 5, right column). Additionally, the excitatory response areas were 
PRESYNAPTIC

POSTSYNAPTIC
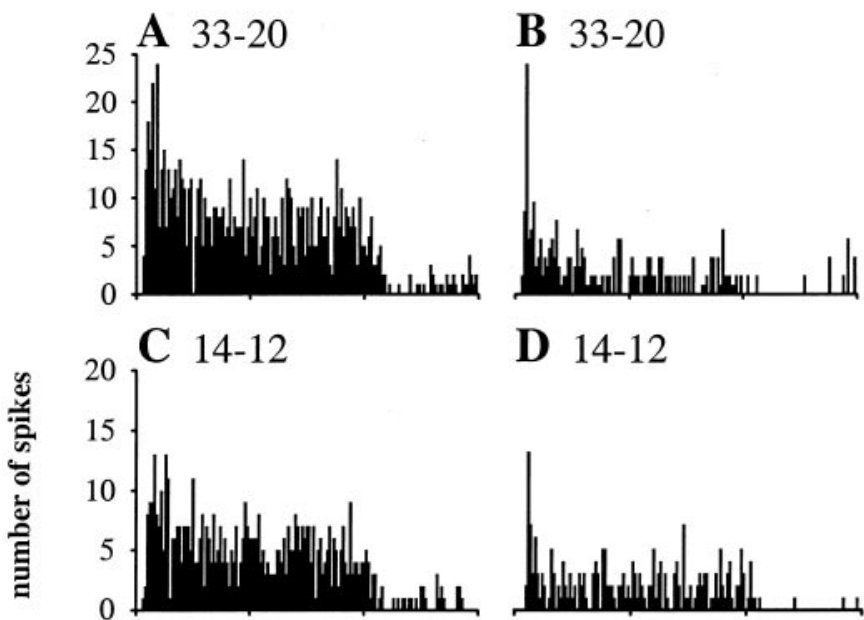

D $14-12$
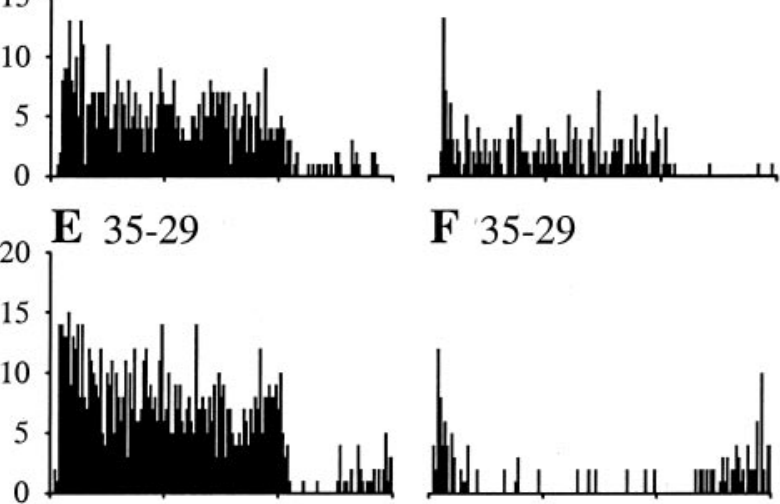

F $35-29$
PREDRUG

DRUG APPLICATION
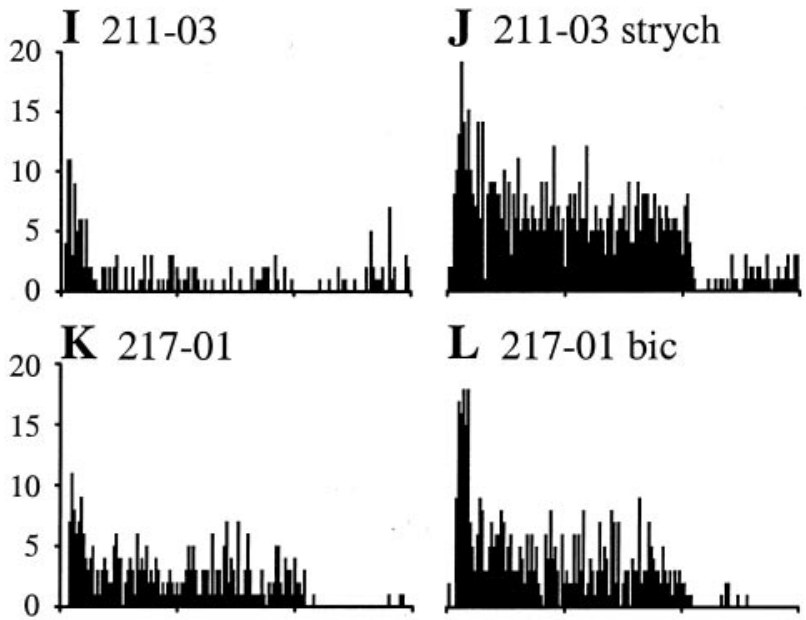

L 217-01 bic
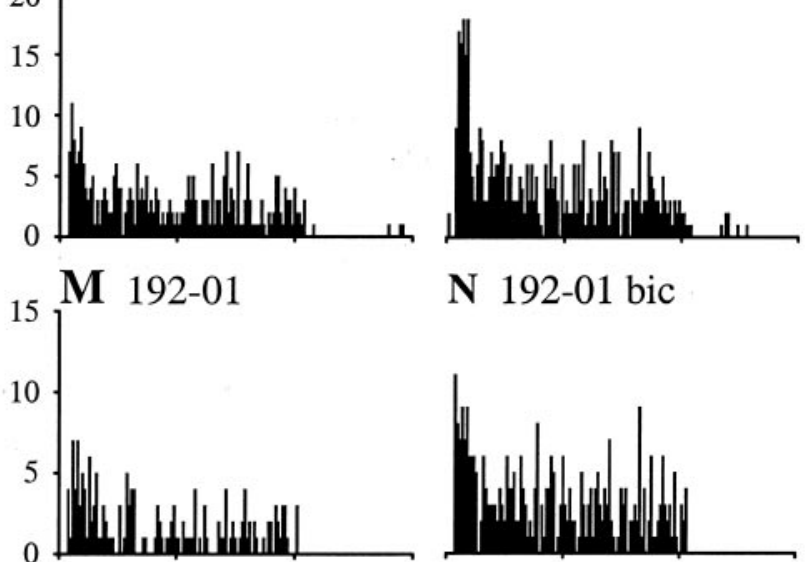

N $192-01$ bic

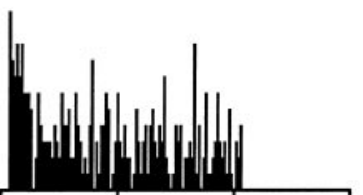

SUMMED PSTH
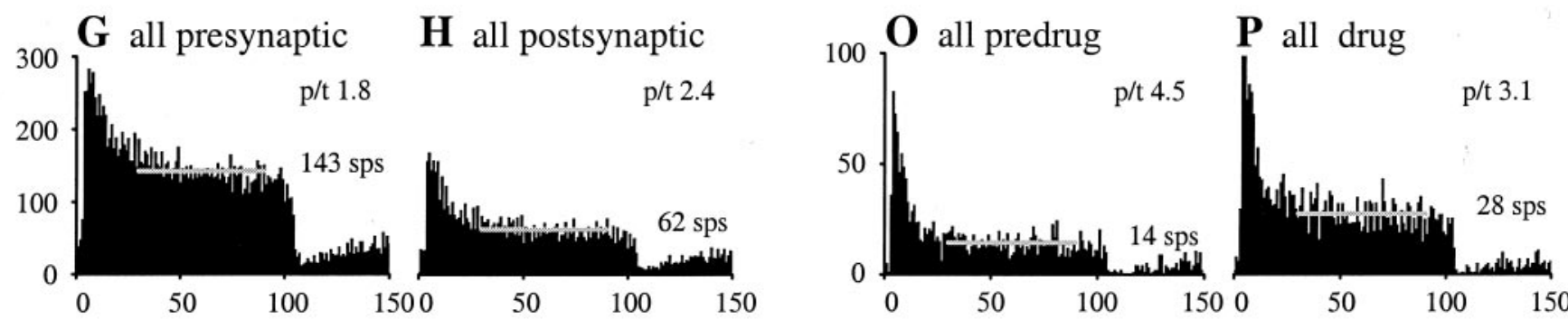

time [msec]

Figure 3. PSTHs in six primary-like units. Tone bursts of $100 \mathrm{msec}$ at the CFs of the units were presented with $80-90 \mathrm{~dB}$ SPL; bin width $=1 \mathrm{msec}$. $A$, $C, E$, PSTHs of presynaptic recordings compared with postsynaptic PSTHs of the respective units $(B, D, F)$. Predrug PSTHs $(I, K, M)$ compared with PSTHs during drug application $(J, L, N)$. Bottom row shows summed-up PSTHs of 22 units (presynaptic/postsynaptic) $(G, H)$ and 7 units (predrug/drug application) $(O, P)$. Average steady-state rates $(30-90 \mathrm{msec})$ are indicated as gray lines and given as sps; peak-over-total values $(p / t)$ are given in the top right corner of each PSTH. strych, Strychnine; bic, bicuculline.

restricted at the low-frequency flank (Fig. 5A) [in other cases also at the high-frequency flank (data not shown)] or toward higher stimulus levels (Fig. 5B). In both examples, the reduced postsynaptic discharge activity revealed inhibition that is tuned to the neurons' excitatory response areas near CF. This will further be referred to as "on-CF" inhibition.

To quantify the difference in presynaptic and postsynaptic frequency selectivity, the difference in bandwidth was calculated in $10 \mathrm{~dB}$ steps between 10 and $60 \mathrm{~dB}$ above the thresholds of the units. Presynaptic and (in most cases) also the postsynaptic band- width increased with level. However, comparison revealed a significant presynaptic to postsynaptic increase in frequency selectivity (Fig. 6). This increase was small at $10 \mathrm{~dB}$ above threshold and became progressively larger at higher stimulus levels.

To test whether inhibition shapes postsynaptic response areas, we applied strychnine or bicuculline iontophoretically. The unit shown in Figure $7 A$ had an excitatory postsynaptic response area restricted to a circumscribed frequency/intensity domain between 1.0 and $2.0 \mathrm{kHz}$ and between 20 and $40 \mathrm{~dB}$ SPL. At higher intensities firing was reduced and even fell below the SR, reveal- 


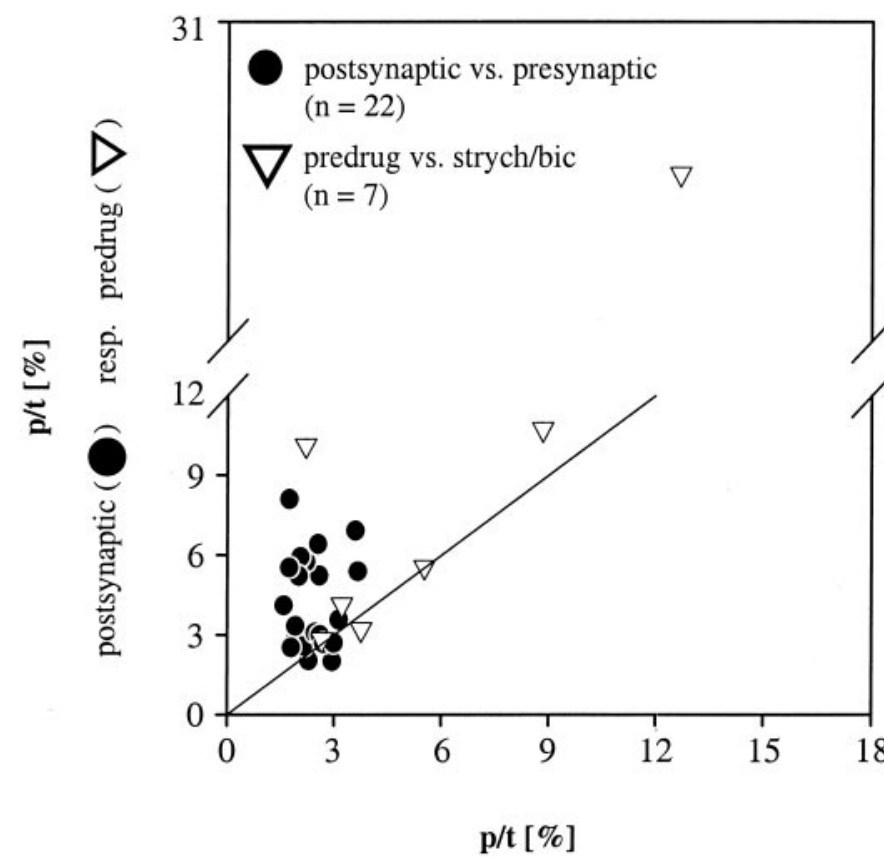

presynaptic $(\bigcirc) \quad$ resp. $\operatorname{strych} /$ bic $(\nabla)$

Figure 4. Peak-over-total ratios $(p / t)$ of the PSTHs for postsynaptic versus presynaptic $(\bullet)$ response, respectively, predrug versus drug application $(\triangle)$. $P / t$ was calculated as the number of spikes in the first $1 \mathrm{msec}$ bin expressed as a percentage of the total number of spikes in the first 100 $\mathrm{msec}$ of the response.

ing an inhibitory area that extended over the whole frequency range tested (Fig. 7A1,2) (see Fig. $5 B$ for a PP unit with a comparable upper threshold). The effect of inhibition is also seen in the nonmonotonic course of the rate-level function (Fig. 7A3). This type of inhibition, which covers a broad frequency range, will further be referred to as "broadband inhibition." After the application of strychnine, the postsynaptic excitatory response area extended to higher intensities and disclosed an area of excitation as typically seen for the presynaptic activity (compare Fig. 5B1,2 with Fig. 7B1,2). Blocking glycinergic inhibition caused an increase in discharge rate as indicated by the rate-level function (Fig. 7B3). SR increased from 117 to 149 sps, and discharge rates at $50 \mathrm{~dB}$ SPL CF stimulation increased from 103 to 312 sps. The rate-level function took a monotonic course. The strychnine application mostly caused a block of inhibition while leaving the sidebands. The recovery shown in Figure $7 C$ indicates that this strychnine block was reversible.

A second example shows the response of another unit to the application of bicuculline (Fig. 7D,E). Blocking GABAergic inhibition causes an increase in both spontaneous (from 8 to $39 \mathrm{sps}$ ) and sound-evoked discharge rate (from 69 to $151 \mathrm{sps}$ ) (Fig. $7 D 1, E 1)$. Still, the frequency selectivity of this unit was not influenced by inhibition, pointing to an on-CF type of inhibition (Fig. 7D2,E2). The increase in discharge rate is also reflected in the rate-level functions (Fig. 7D3,E3).

If all units that underwent drug application were regarded, differences in the frequency tuning occurred exclusively in units with very strong broadband inhibition and with upper thresholds (4 of 19) when glycinergic inhibition was blocked (2 of 4) but not when GABAergic inhibition was blocked ( 2 of 4$)$.

\section{Rate-level functions}

The presynaptic to postsynaptic reduction in discharge rate also becomes evident from a comparison of the rate-level functions (Figs. 5A,B, right column, 8). All presynaptic recordings had monotonic rate-level functions with a mean dynamic range of $35 \pm 10 \mathrm{~dB}(n=22)$. The dynamic ranges were regarded as the difference between the response thresholds of the units and the levels evoking maximal discharge activity. In contrast, only approximately half of the postsynaptic recordings exhibited monotonic rate-level functions (13 of 22) (Fig. 8A). In the latter, the mean dynamic range was $46 \pm 14 \mathrm{~dB}$ and thus significantly larger than the respective presynaptic values $(37 \pm 10 \mathrm{~dB} ; n=13$; paired $t$ test; $p=0.05)$. The remaining units had nonmonotonic postsynaptic rate-level functions [20\% decrease from maximum as defined in Winter and Palmer (1990)] (Figs. 5A6,B6, 8B), with a mean dynamic range of $31 \pm 11 \mathrm{~dB}(n=9)$. This was not significantly different from the respective presynaptic dynamic range of $33 \pm 11 \mathrm{~dB}$ (paired $t$ test; $p=0.66$ ). In the pharmacological experiments, units with monotonic rate-level functions (four of nine) showed an increase in discharge rates when the inhibition was blocked (Fig. $8 C$ ). In the nonmonotonic units (five of nine) (Fig. 8D), the block of inhibition caused the rate-level functions to take a monotonic course (both blocked with strychnine) in two cases. The remaining three units (two bicuculline, one strychnine) showed an overall increase in discharge rates, but the units kept their nonmonotonic course. In units with nonmonotonic as well as monotonic rate-level functions, the difference between presynaptic and postsynaptic activity or predrug and drug activity increased with stimulus intensity (Fig. 8, right column), pointing to an increased influence of inhibition at higher intensities.

If inhibition causes the formation of coherent areas with reduced postsynaptic activity, then the inhibition should also be reflected in a higher proportion of isolated PPs in these domains. The calculation of the spike failure could thus be used to describe and quantify inhibitory response areas and thereby provide an alternative way to visualize the tuning and dynamics of inhibitory influence. In Figure 9, this analysis is shown for two representative units that show the predominant types of inhibition, on-CF inhibition (078-14, top row) and broadband inhibition (078-13, bottom row). The presynaptic response areas of both units resemble those of ANFs (Fig. 9A,D). Unit 078-14 had an excitatory response area with an upper threshold (Fig. 9B). The on-CF inhibition is here visualized by frequency/intensity domains with high spike failure rates (Fig. 9C). The unit 078-13 had prominent broadband inhibition that was reflected in the inhibitory sidebands (Fig. 9E) and in the frequency/intensity domains with high spike failure rates (Fig. 9F). For both units the strength of inhibition increased with intensity.

\section{Responses to two-tone stimulation}

From the pharmacological data presented above and from the analysis of spike failures, we conclude that the differences between presynaptic and postsynaptic activity at the ANF-SBC synapse are caused at least in part by neuronal inhibition. In extracellular recordings, an inhibitory influence can be manifested only as a reduction in the spontaneous or sound-evoked activity of a neuron. Here, we used two-tone stimulation (Fig. 10) in a series of experiments to reliably confirm the occurrence of acoustically evoked inhibition in PP units regardless of their different SRs. A probe-tone $(100 \mathrm{msec})$ was set at the CF of the unit and presented at intensities $\geq 20 \mathrm{~dB}$ above threshold to 

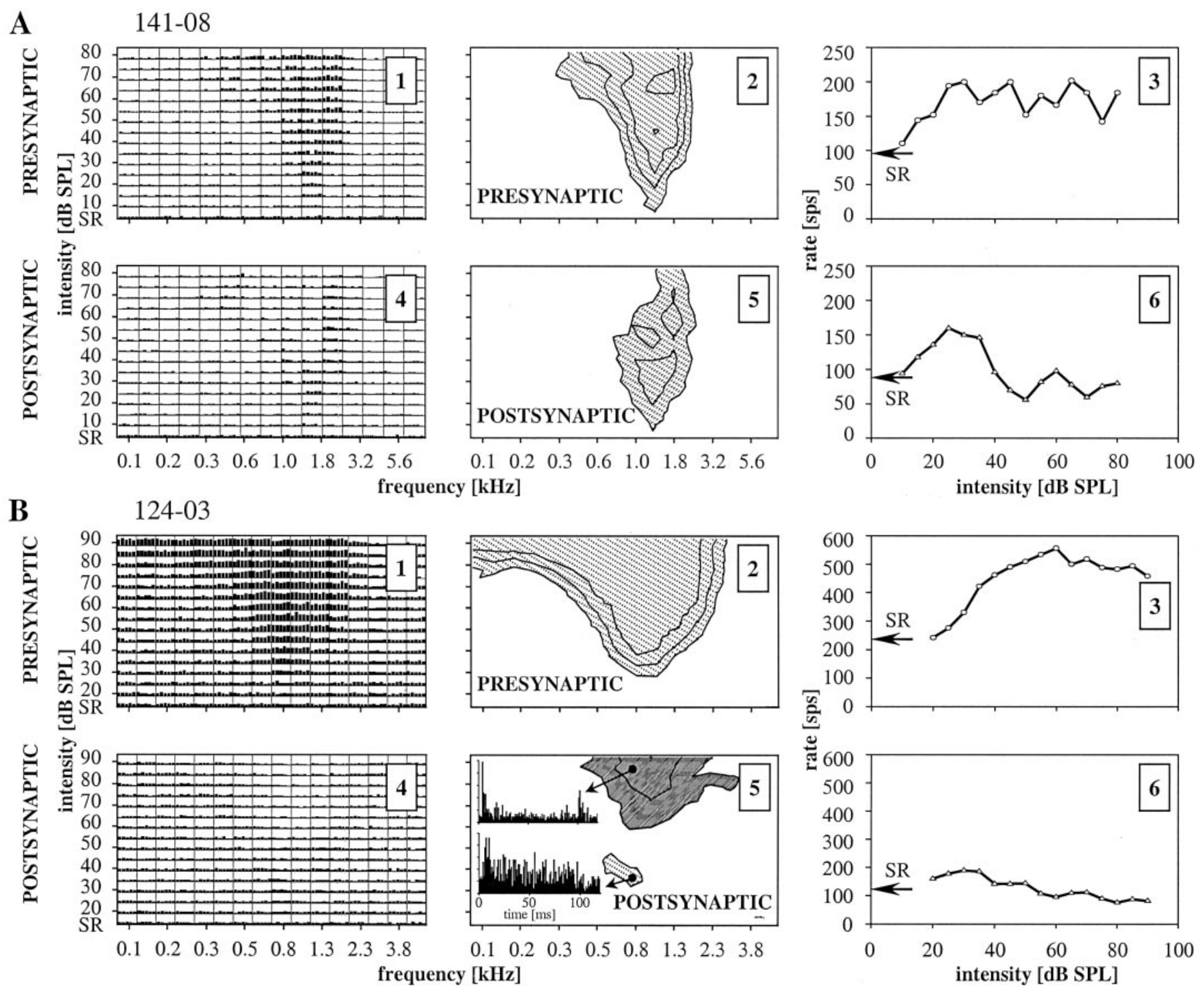

Figure 5. Comparison of presynaptic (top panels) and postsynaptic (bottom panels) excitatory response areas. For stimulation protocol refer to Materials and Methods. $A 1,4, B 1,4$, Spike rates evoked by a single stimulus are indicated by the heights of the bars. A2,5, B2,5, Iso-response contours calculated from the response area shown on the left. For details see Materials and Methods. $A$, Unit $141-08$; CF, $1.3 \mathrm{kHz}$. Postsynaptically the excitatory response area is narrower at stimulus levels above $50 \mathrm{~dB}$ SPL. $B$, Unit $124-03$; CF, $0.8 \mathrm{kHz}$. Here, the postsynaptic excitation is reduced to a small circumscribed response area (B5, dotted area). At stimulus levels above $50 \mathrm{~dB}$ SPL, a prominent area of inhibition is seen (hatched area) where the presynaptic recording displays excitation. B5, Insets, Primary-like (bottom) and on-off (top) PSTHs within the postsynaptic response area. A3,6, B3,6, Rate-level functions of presynaptic and postsynaptic recordings; $S R$ is indicated by arrows.

induce varying levels of stimulus-evoked, "steady" activity (compare Fig. 5B1,2), which allows inhibitory effects of a test-tone to be detected as a reduction of this activity (Fig. 10C,D). The test-tone of $40 \mathrm{msec}$ was delayed by $30 \mathrm{msec}$ relative to the onset of the probe-tone. This allowed the test-tone-induced reduction in activity and the time course of the reduction in activity to be measured [for further details see Kopp-Scheinpflug et al. (2002)]. The latency of inhibition was measured in 10 units and compared with the latency of excitation. For this purpose, the excitatory latency was defined as the time between the stimulus onset and the time at which the neuron reached its half-maximal discharge rate. The inhibitory latency was measured at test-tone frequencies that induced maximal rate reduction and was defined as the time between the onset of the test-tone and the time at which the tonic component of the response of the neuron was reduced to its half-maximal level. On average, inhibitory latencies $[>50 \mathrm{~dB}$ SPL; median, $6.1(4.9,9.0) \mathrm{msec}]$ were significantly longer than excitatory latencies [ $>50 \mathrm{~dB}$ SPL; median, $4.5(3.8,5.5)$ msec; $n=$ 10; Wilcoxon signed rank test; $p=0.001$ ].

In many studies it has proven difficult to separate the consequences of cochlear suppression from inhibition because both mechanisms effectively reduce the spike activity of SBCs. In the present two-tone experiments, cochlear suppression will be reflected in both the presynaptic and postsynaptic activity. An inhibitory influence can be revealed from the differences in presynaptic and postsynaptic activity. The example shown in Figure 11 (same unit as in Fig. 5A) illustrates the consequences of suppression and inhibition in distinct high- and low-frequency 


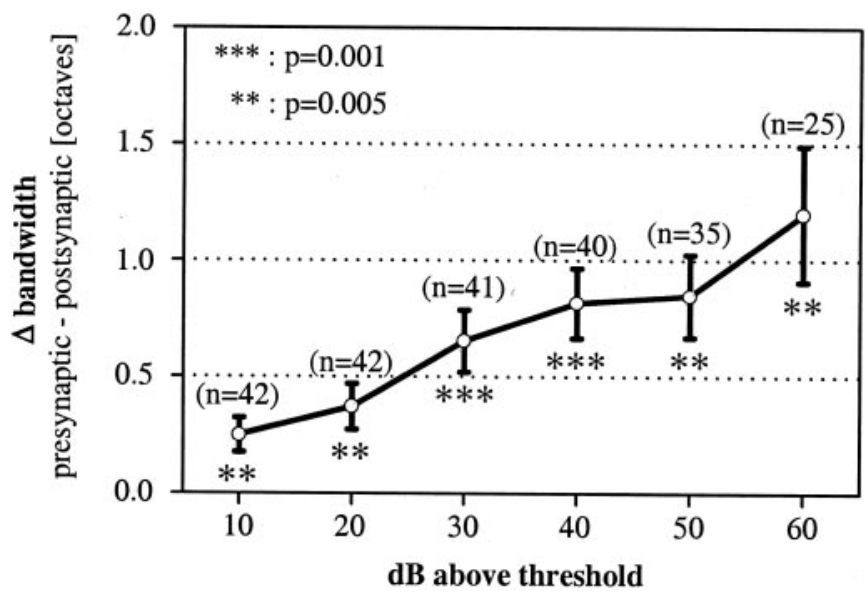

Figure 6. Difference in bandwidth between presynaptic and postsynaptic activity increases with stimulus level. All values (presynaptic vs postsynaptic bandwidth) differed significantly ( $t$ test; $p$ value indicated). Because of the different threshold values of the units, the number of tested pairs $(n)$ decreased toward higher levels.

sidebands flanking the excitatory response area. At probe-tone levels of 10 and $20 \mathrm{~dB}$ above threshold, the presynaptic and postsynaptic recordings showed distinct high-frequency suppression sidebands that overlapped only slightly with the excitatory response areas. These suppression sidebands were reduced and finally disappeared at higher probe-tone levels (Fig. 11B,C). Postsynaptically, the high-frequency sidebands covered a somewhat wider area, pointing to an additional influence of inhibition. The influence of postsynaptic inhibition is clearly seen in the low-frequency sidebands (Fig. $11 D-F$ ), which have no presynaptic correspondence (Fig. $11 A-C$ ).

Inhibition of SBC spiking rather than suppression is indicated when the test-tone-induced reduction of the probe-tone response was greater postsynaptically than presynaptically. To measure the differential effect of the test-tones on presynaptic and postsynaptic activity, the failures of postsynaptic spike generation were compared in 42 PP units under three different conditions: (1) no stimulation (SR), (2) single-tone stimulation (at CF), and (3) two-tone stimulation (test-tone presented outside the presynaptic and postsynaptic excitatory response areas of the unit, at high probe-tone intensities). As noted above, the mean failure rates were $42 \pm 27 \%$ for the SR. After probe-tone stimulation, both the presynaptic and postsynaptic activity increased, but to a different extent, resulting in a significant change in the failure rate $(54 \pm$ $21 \%$ ). Addition of the test-tone caused a noticeable drop in postsynaptic spikes and produced an even greater increase in failure rate $(75 \pm 16 \%)$. The large increase in failure rates during two-tone stimulation provides further evidence for an acoustically evoked inhibition of SBC firing.

For the same unit as in Fig. 11, the presynaptic and postsynaptic differences in the responses to two-tone stimulation can also be seen in the "iso-intensity curves" (Fig. 12A,B). For recording of the iso-intensity curves, probe-tones were presented at $\mathrm{CF}$ and $20 \mathrm{~dB}$ above threshold, and the test-tone was systematically varied in frequency at a single fixed intensity for each of the curves. The presynaptic recording yielded reductions of the discharge activity mostly in a frequency band above CF (Fig. 12A). Postsynaptically, prominent high- and low-frequency sidebands are visible also at lower test-tone intensities (Fig. 12B). During two-tone stimula- tion, 23 of 42 units showed signs of suppression in the presynaptic response (Fig. 12C,E), and for 38 of 42 units, significant inhibitory influences were observed in the postsynaptic responses (Fig. $12 D, F)$. In 27 units of this sample, prominent inhibitory sidebands flanked and partly overlapped the postsynaptic excitatory response areas (broadband inhibition) (Fig. 12D), whereas in the remaining 11 units, the inhibitory areas covered high intensities within the excitatory response areas of the units, including the CFs of the units (on-CF inhibition) (Fig. 12F).

\section{DISCUSSION}

SBCs in the AVCN receive input through large calyceal endings that has long been thought to primarily determine SBC firing. By simultaneously recording from the presynaptic and postsynaptic neurons, it is possible to assess directly to what extent presynaptic and postsynaptic firing are correlated. The present recordings indicate that firing in the endbulb often fails to cause postsynaptic spikes.

\section{Analysis of waveforms}

Pfeiffer (1966a) first interpreted the complex waveforms in the AVCN and introduced the term prepotential for the presynaptic components of these signal complexes. In the present study we argue that spike-preceding PPs and isolated PPs represent the firing of the same endbulb. This interpretation is based on the results of the principal component analysis and on a number of common properties of the two signals. Slight differences in shape and amplitude are seen occasionally between spike-preceding PPs and isolated PPs (Fig. 1). However, similar differences also occur between postsynaptic spikes of a single unit and thus are not inconsistent with our interpretation. This variability is likely attributable to the conditions of extracellular recording techniques in vivo.

\section{Spike failure}

Previous electrophysiological studies of the AVCN in which PP units were documented considered only their postsynaptic response (Shofner and Young, 1985; Young et al., 1988; Winter and Palmer, 1990; Winter et al., 1990). In the present study, we examined the presynaptic response as well. Our presynaptic and postsynaptic SRs are comparable with what was reported for low-frequency ANFs and SBCs (Blackburn and Sachs, 1989; Schmiedt, 1989; Ohlemiller and Echteler, 1990; Spirou et al., 1990; Smith et al., 1993; Müller, 1996). However, the difference that we found between presynaptic and postsynaptic discharge rates indicates failures in spike transmission at this synapse, which cannot be seen when comparing unrelated populations of ANFs and SBCs. The change in the SR during drug application was smaller than the difference between presynaptic and postsynaptic $\mathrm{SR}$, which indicates that cellular mechanisms in addition to GABAergic or glycinergic inhibition also affected postsynaptic discharges (Rothman et al., 1993). Still, the increase in failure rate from spontaneous to two-tone-driven activity is in accordance with Pfeiffer's early reports (1966a), in which he estimated that failures of postsynaptic spikes ("B component") occurred in approximately one-quarter of the units and claimed "that the B component can be made to fail more frequently, for a given neuron, by increasing the sound pressure level of acoustic stimuli."

\section{Inhibition in SBCs}

Earlier reports are unclear about the occurrence of acoustically evoked inhibition on SBCs. Sideband stimulation did not decrease the SR of the units (Goldberg and Brownell, 1973), and 

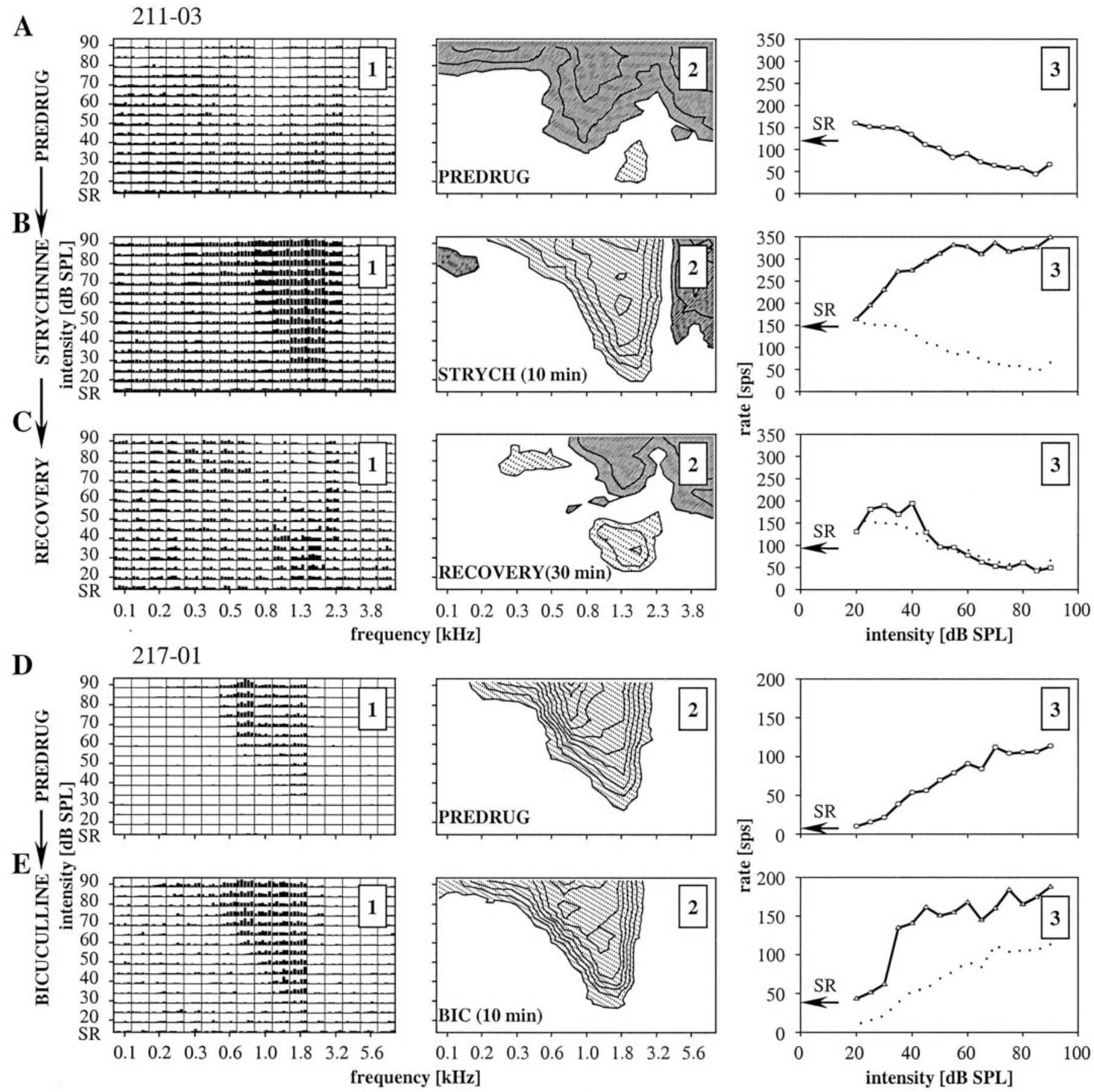

Figure 7. Pharmacological block of glycinergic and GABAergic inhibition in two SBCs. The design of the graph is the same as Figure 5. Dotted lines in $B 3, C 3$, and $E 3$ indicate the predrug rate-level functions. $A-C$, Unit $211-03$ : predrug condition $(A)$; strychnine application $(B)(50 \mathrm{nA} / 3$ min); recovery $(C)$ (current of $-15 \mathrm{nA}$ for $30 \mathrm{~min}$ ). $D, E$, Unit 217-01: predrug condition $(D)$; bicuculline application $(E)(25 \mathrm{nA} / 10 \mathrm{~min})$. STRYCH, Strychnine; BIC, bicuculline.

two-tone stimulation could not clearly distinguish suppression from inhibition (Rhode and Greenberg, 1994). Several factors must be considered when extracellular recording is used to study inhibition. First, inhibition can be measured only in spiking neurons. Second, excitation and inhibition are nonlinearly related to each other. Thus, estimates of the contribution of inhibition depend on the analysis of multiple recordings in which the levels of excitatory and inhibitory stimuli are varied independently. Third, in the auditory system, sideband suppression and neuronal inhibition both reduce firing, but through different mechanisms. Suppression arises from the mechanics of the cochlea during two-tone stimulation. The discharge rate of an ANF elicited by moderate-level pure-tone stimuli at the CF of the fiber can be reduced by a second tone at neighboring frequencies, which does not affect the discharge rate of the fiber when it is presented alone (Galambos and Davis, 1943; Sachs and Kiang, 1968). Consequently, any stimulus-induced rate reduction of AVCN neurons could result from inhibition or two-tone suppression or both 


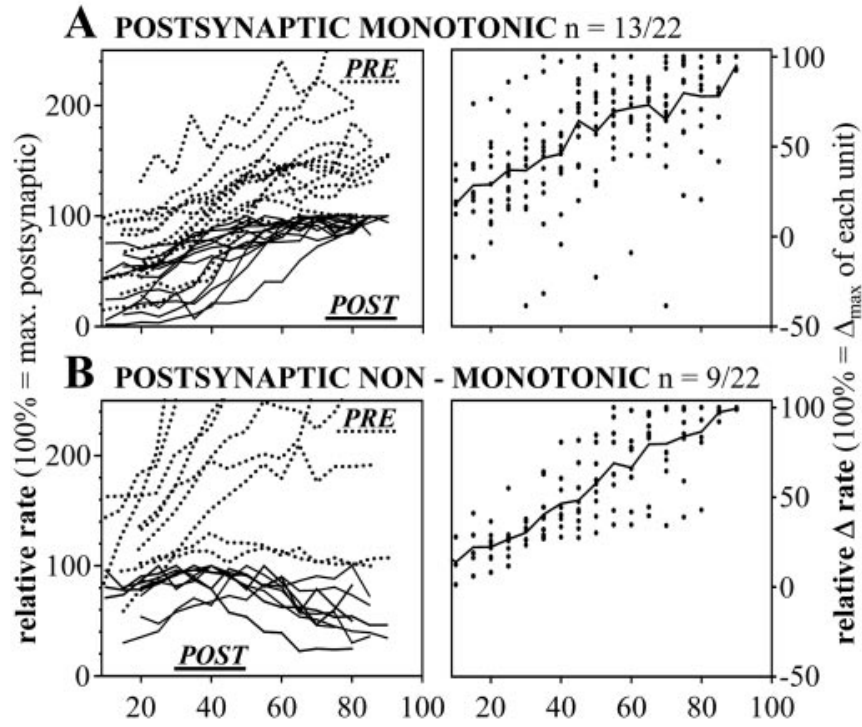

C Predrug monotonic $\mathrm{n}=4 / 9$

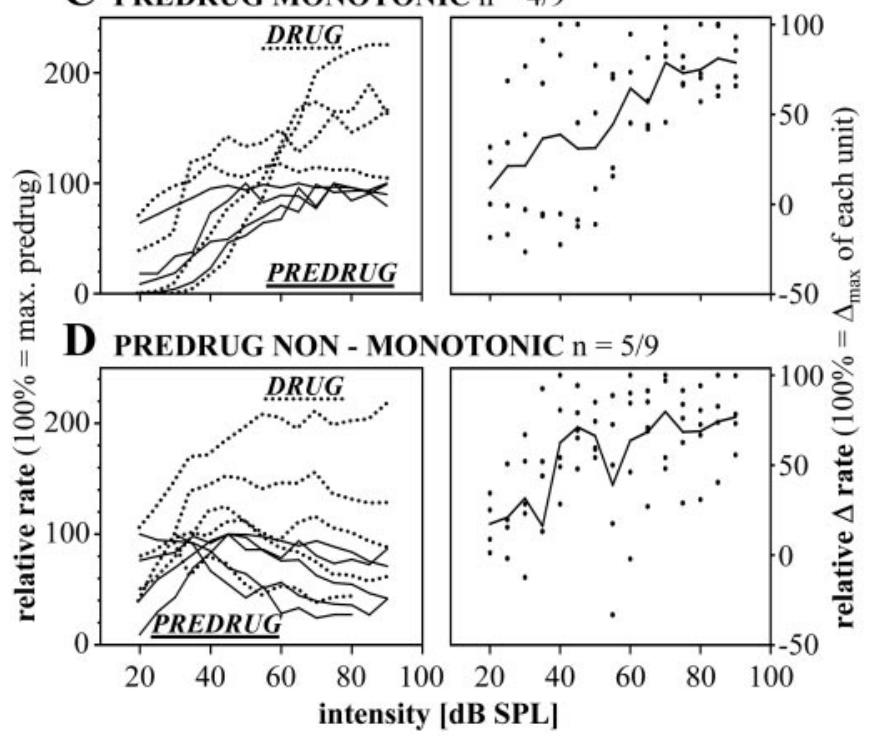

Figure 8. Rate-level functions of units with presynaptic $(P R E)$ and postsynaptic $(P O S T)$ recordings $(A, B)(n=22)$ or recordings before $(P R E D R U G)$ and during $(D R U G)$ drug application $(C, D)(\mathrm{n}=9)$. Rate-level functions were averaged for the CF and two neighboring frequencies. Left column, Each function was normalized to the maximum discharge rate of the postsynaptic or predrug response, respectively (black lines), and compared with the presynaptic or drug response (dotted lines). $A, C$, Postsynaptic response predrug monotonic rate-level functions; $B, D$, nonmonotonic rate-level functions. $B$, Four of the nine units showed an increase in presynaptic discharge rate that exceeded postsynaptic rate by a factor of 2.5. Right column, Differences between presynaptic and postsynaptic responses, respectively, predrug and drug responses of the absolute count data, normalized to the maximum difference of each unit $(100 \%)$. Values for single units are symbolized by dots; solid lines indicate the running averages.

processes. In our approach, suppression (indicated in the presynaptic activity) can be eliminated by adjusting the probe-tone intensity, so that the remaining postsynaptic rate reduction can be attributed to inhibition or other cellular mechanisms (e.g., intrinsic membrane properties of SBCs) (Manis and Marx 1991). In the present study, the contribution of inhibition was verified by pharmacological experiments.

\section{Frequency selectivity}

The shape and bandwidth of presynaptic and predrug frequency threshold curves were comparable with those of ANFs (Schmiedt, 1989; Ohlemiller and Echteler, 1990; Müller, 1996). Postsynaptic response areas showed sharper frequency selectivity and more variability with respect to shape and threshold. In neuropharmacological experiments, Caspary et al. (1994) found that AVCN primary-like units had so-called broad or near-CF inhibition, similar to the types of inhibition that we observed. However, the reduction in frequency selectivity after a block of inhibition differed in our respective experiments. Caspary et al. (1994) found a loss of sharpness after drug application of $\sim 0.2$ octaves, measured $25-35 \mathrm{~dB}$ above the thresholds of the units, whereas at this level we observed a loss of sharpness of $\sim 0.6$ octaves. As shown in the present study, the contribution of inhibition to the sharpening of postsynaptic tuning increases with intensity, suggesting that different threshold criteria might underlie this difference in the magnitude of sharpening. Caspary et al. (1994) made the qualification that, because of the limited ranges of frequencies tested, they might have missed some contribution of lateral inhibition or of "very" broad inhibition. Thus, the differences between experiments might also be attributable to the different experimental protocols. Although both pharmacological studies provide strong evidence for the effectiveness of GABAergic and glycinergic inhibition on sound-induced SBC activity, this inhibition may not account completely for the differences between presynaptic and postsynaptic activities. Additional factors might include other inhibitory transmitter systems, incomplete block of inhibition, or intrinsic membrane properties.

To our knowledge, the present study is the first that reports broadly tuned and very strong inhibitory input on SBCs that greatly increases their frequency selectivity (Figs. 5B, 7).

\section{Rate-level functions}

The presynaptic recordings had monotonic rate-level functions, as described for the ANFs (Winter et al., 1990; Ohlemiller et al., 1991). The postsynaptic occurrence of nonmonotonic rate-level functions in SBCs (Figs. 5, 7, 8) is in accordance with the assumption of "center-band inhibitory effects" suggested by Winter and Palmer (1990). Here, we showed that nonmonotonic rate-level functions in SBCs can be caused by glycinergic on-CF inhibition. However, there were also units (three of five) in which the block of inhibition did not cause a change from nonmonotonic to monotonic rate-level functions. This might have been because of incomplete block of glycinergic inhibition. Alternatively, these units could have received an additional GABAergic inhibition that was not blocked. Although we did not test bicuculline and strychnine simultaneously or consecutively for these units (Fig. $7 A-C)$ with extreme broadband inhibition $(n=4$; two strychnine, two bicuculline), the results might imply that glycinergic inhibition rather than GABAergic inhibition induces upper thresholds and nonmonotonic rate-level functions. Although the data provide evidence that inhibition alters postsynaptic rate-level functions, the possibility that intrinsic properties of the SBCs might also contribute to nonmonotonic rate-level functions at high levels of excitatory synaptic input cannot be excluded (Carney, 2002).

\section{Response patterns}

In the presynaptic recordings, all units showed the typical PSTHs described for ANFs (Schmiedt, 1989; Müller, 1996). In our 

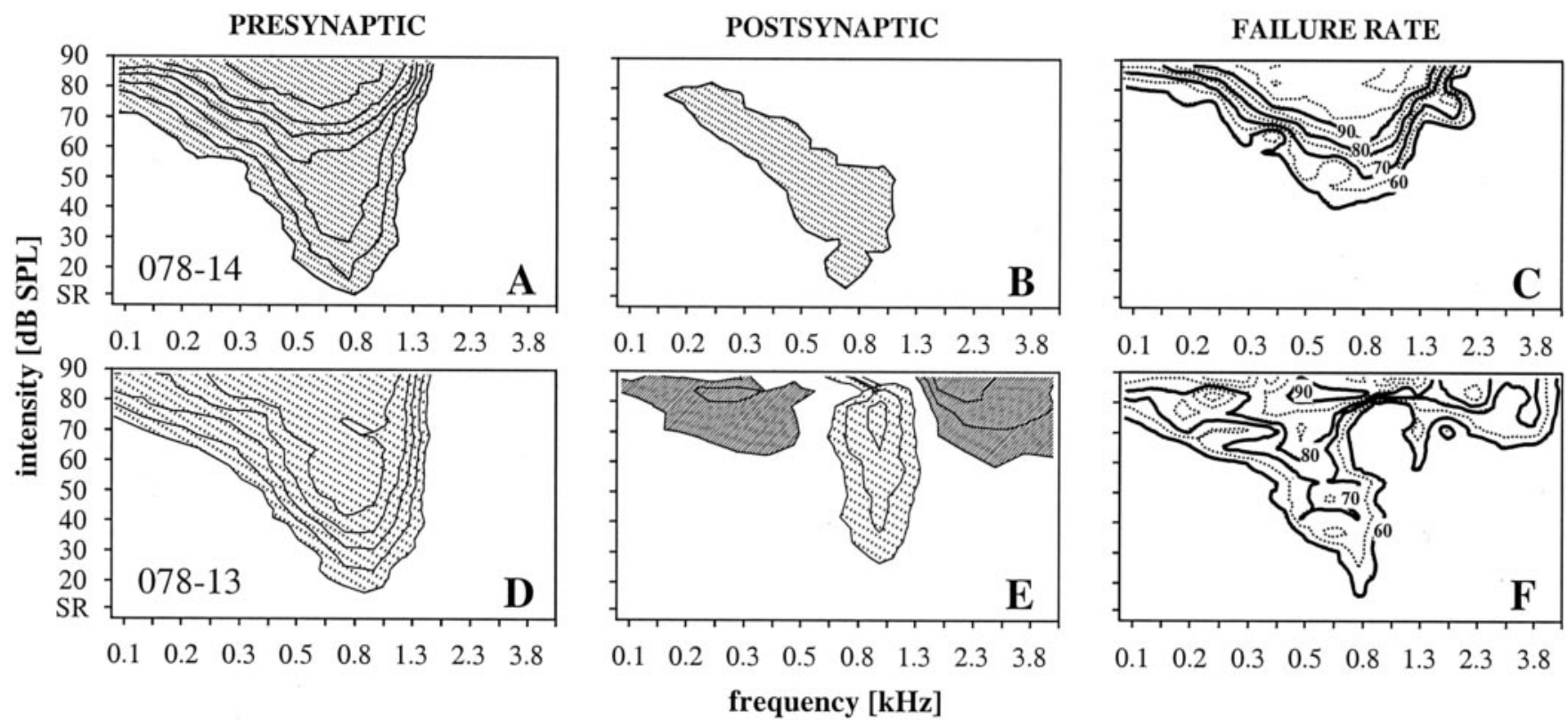

Figure 9. Differences between presynaptic and postsynaptic response areas related to the occurrence of isolated PPs. $A-C$, Unit $078-14 ; D-F$, unit 078-13; presynaptic $(A, D)$ and postsynaptic $(B, E)$ excitatory response areas. In $E$ the excitatory response area (dotted) is enclosed by inhibitory sidebands (hatched). $C, F$, Occurrence of isolated PPs indicated as iso-contours of percentage failure rates $(60-90 \%)$.

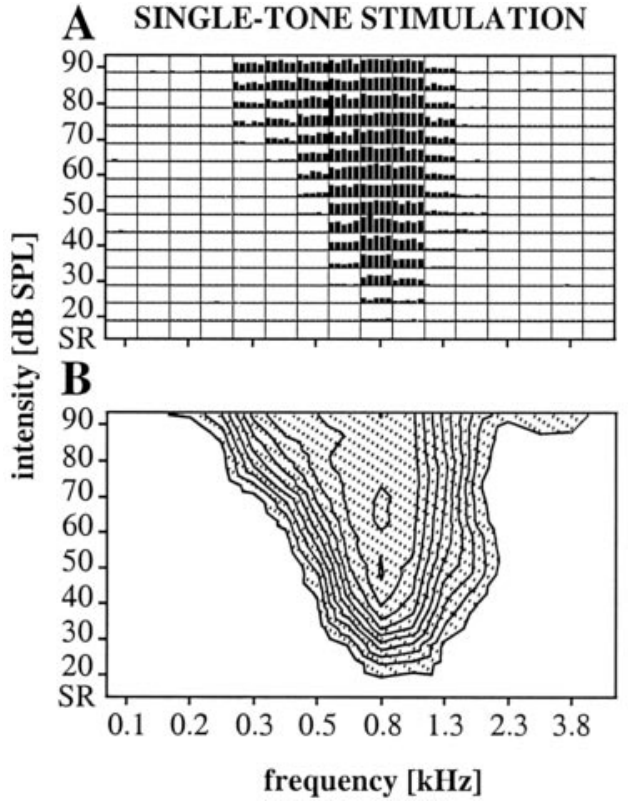

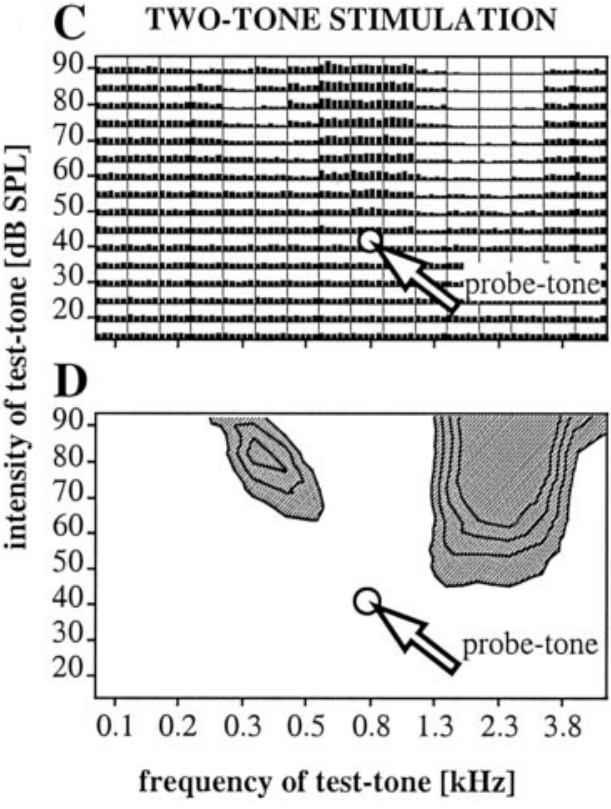

Figure 10. A, Excitatory frequency/intensity response area (design of the graph is same as Fig. 5). This unit had virtually no SR (bottom row). $C$, Frequency/intensity response area during two-tone stimulation (probe-tone: $\mathrm{CF} / 20 \mathrm{~dB}$ above threshold; test-tones varied in the frequency/intensity range indicated by the matrix). The shown spike rates were evaluated over the 40 msec test-tone periods (i.e., 30-70 msec). The probe-tones generated a constant amount of excitation (indicated by equal bar heights in bottom row). In two frequency/intensity domains (flanking the excitatory response area shown in $A$ ), the probetone-induced discharges were reduced by the test-tones. $B, D$, Iso-response contours calculated from the response areas shown in $A$ and $C ; B$, dotted: increased activity above SRs; $D$, hatched: activity reduced below probe-tone-induced discharge rates. postsynaptic recordings, $\mathrm{SBCs}$ (at $\mathrm{CF} /$ near threshold) showed the same primary-like PSTH (Pfeiffer, 1966b). At higher levels, however, some SBCs responded predominantly at stimulus onset or offset, or both, and these responses were always associated with on-CF inhibitory areas. As quantified by the width of the peak and the peak-over-total ratio, the on-CF inhibition reduced firing after the first sound-evoked postsynaptic spike and thereby shortened and enhanced the onset component in the PSTH relative to the steady state. The stronger reduction of the steady state compared with the onset can be explained by an inhibition that is delayed relative to the excitation, as shown in the present study.

\section{Possible sources of inhibition}

The present results show that the morphologically homogeneous population of $\mathrm{SBC}$ s receives different types of inhibition (on-CF or broadband) and thus might serve different functions in auditory brainstem processing. An SBC might be classified as receiving either one or even both types of inhibition, depending on the stimulus condition; i.e., only on-CF inhibition is seen during pure-tone stimulation, whereas additional broadband inhibition can be detected in the same unit during two-tone stimulation. For glycinergic inhibition, two major sources have been suggested within the cochlear nucleus: on-frequency inhibition from the 


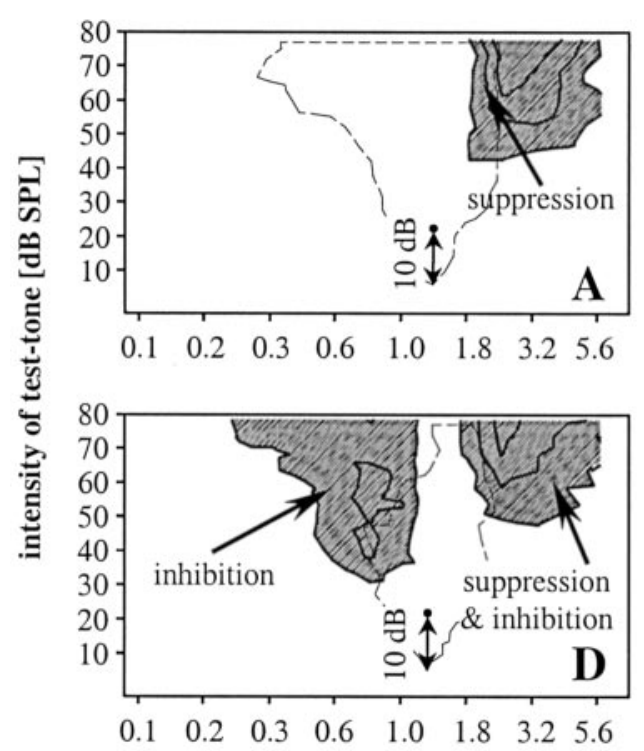

PRESYNAPTIC ACTIVITY

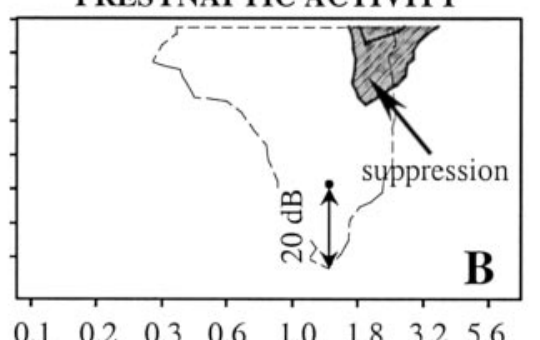

POSTSYNAPTIC ACTIVITY

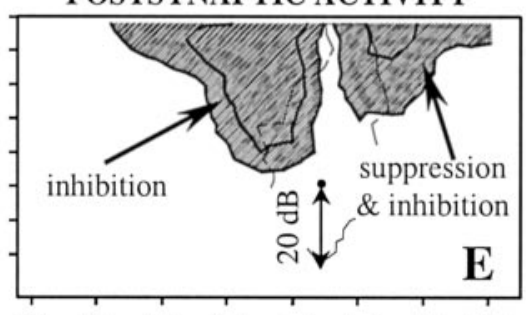

$\begin{array}{llllllll}0.1 & 0.2 & 0.3 & 0.6 & 1.0 & 1.8 & 3.2 & 5.6\end{array}$

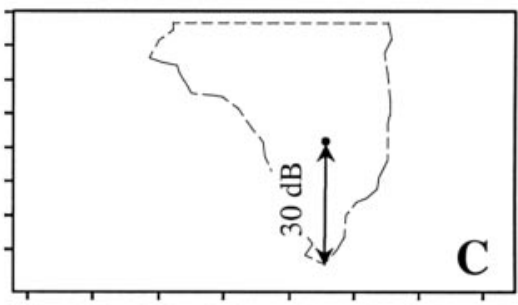

$\begin{array}{llllllll}0.1 & 0.2 & 0.3 & 0.6 & 1.0 & 1.8 & 3.2 & 5.6\end{array}$

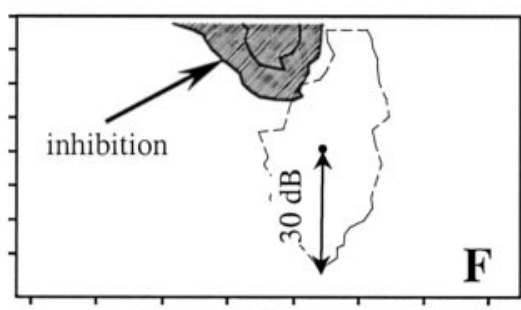

$\begin{array}{llllllll}0.1 & 0.2 & 0.3 & 0.6 & 1.0 & 1.8 & 3.2 & 5.6\end{array}$

frequency of test-tone $[\mathrm{kHz}]$

Figure 11. Distinction between two-tone suppression and neuronal inhibition. All recordings are from unit 141-08. Dashed lines indicate tuning curves for single tone-burst stimulation (for the same unit also shown in Fig. 5A2,5). Here, comparable with Figure $9 E$, the maps show the test-tone-induced reduction of the probe-tone-evoked discharges. The probe tone $($ dot $)$ was presented at the $\mathrm{CF}(1.3 \mathrm{kHz})$ at three levels: $10 \mathrm{~dB}$ above threshold $(A, D)$, $20 \mathrm{~dB}$ above threshold $(B, E)$, and $30 \mathrm{~dB}$ above threshold $(C, F)$.
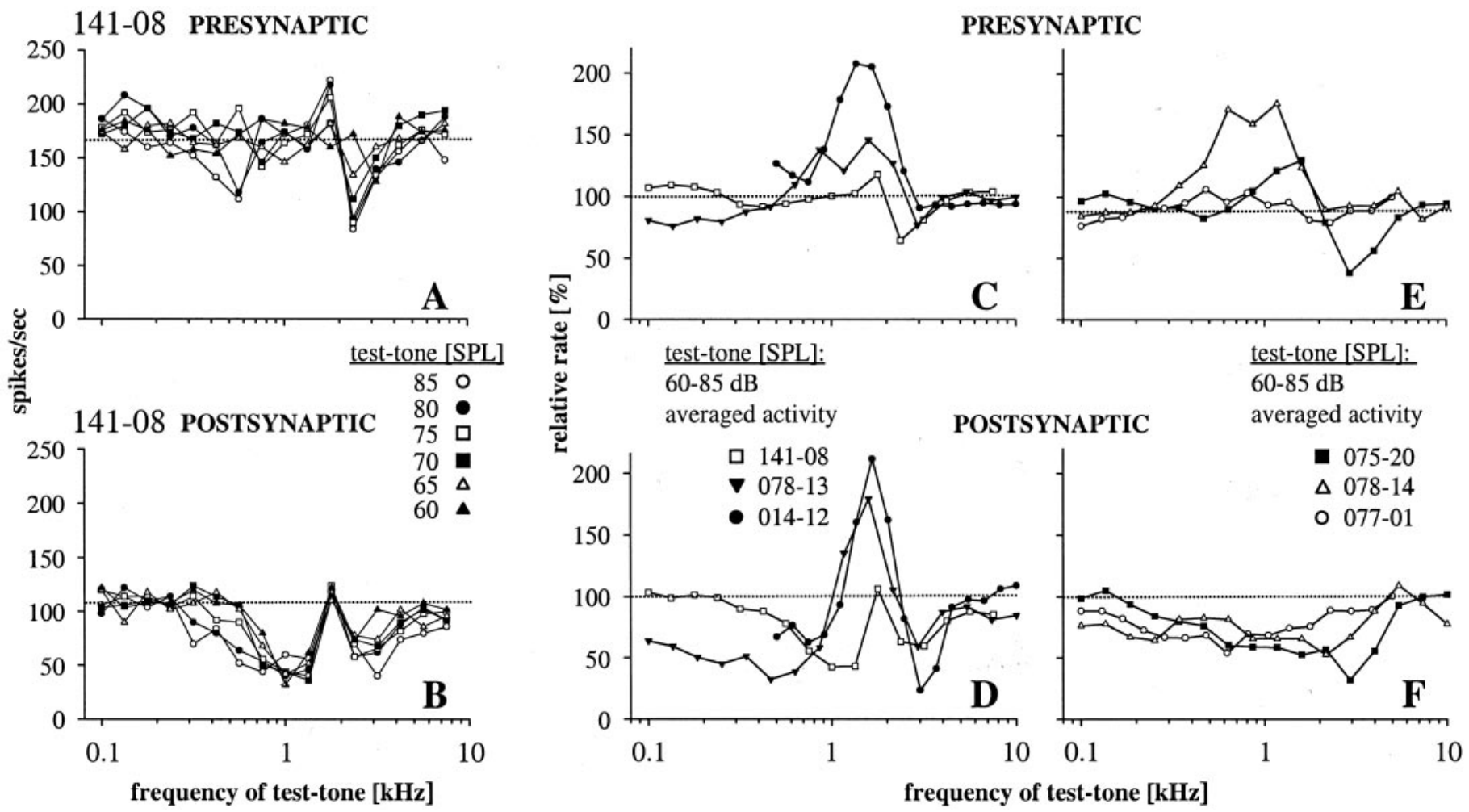

Figure 12. Iso-intensity curves during two-tone stimulation. $A, B$, Respective presynaptic and postsynaptic iso-intensity curves for a $\mathrm{PP}$ unit; $\mathrm{CF}$, 1.3 $\mathrm{kHz}$; probe-tone, $\mathrm{CF} / 30 \mathrm{~dB}$ SPL ( $=20 \mathrm{~dB}$ above threshold); test-tone intensities as indicated in the graph. The dotted lines specify the probe-tone-evoked discharge levels. $C-F$, Presynaptic and postsynaptic iso-intensity curves for six PP units; probe-tones at CF, $20 \mathrm{~dB}$ above threshold. Spike rates are mean values for 60-85 dB SPL test-tone level. The probe-tone-evoked discharges of the respective units were set to $100 \%$. $C, D$, Three units with prominent broadband inhibition in their postsynaptic responses that was not seen presynaptically. $E, F$, Three units with on-CF inhibition. Postsynaptically, the iso-intensity curves show a $25-50 \%$ reduction from the probe-tone-evoked discharge level over a wide frequency range of the response area. 
tuberculoventral cells in the dorsal cochlear nucleus (Young and Voigt, 1982; Wickesberg and Oertel, 1988, 1990; Oertel and Wickesberg, 1993) and broadband inhibition from inhibitory stellate cells within the ventral cochlear nucleus (Smith and Rhode, 1989; Wickesberg and Oertel, 1990; Nelken and Young, 1994; Ferragamo et al., 1998).

In conclusion, our data show that $\mathrm{SBC}$ are the target of acoustically driven inhibition. Inhibition can (1) sharpen the frequency and intensity selectivity, (2) affect the level-dependent dynamic range of responses, and (3) increase the temporal precision of SBC signal onset processing. Whatever additional mechanisms contribute to these effects and how excitation and inhibition are combined in the neuronal networking are not completely understood. Further experiments are required to examine the origin and cellular mechanisms of GABAergic and glycinergic inhibition at the ANF-SBC synapse.

\section{REFERENCES}

Abeles M, Goldstein MH (1977) Multispike train analysis. Proc IEEE 65:762-773.

Adams JC (1981) Heavy metal intensification of DAB-based HRP reaction product. J Histochem Cytochem 29:775.

Bazwinsky I, Härtig W, Rübsamen R (1999) Distribution of different calcium binding proteins in the cochlear nucleus of gerbil and opossum (Abstract 260). Presented at Midwinter Meeting of the Association for Research in Otolaryngology, St. Petersburg Beach, FL, February.

Blackburn CC, Sachs MB (1989) Classification of unit types in the anteroventral cochlear nucleus: PST histograms and regularity analysis. J Neurophysiol 62:1303-1329.

Brawer JR, Morest DK (1975) Relations between auditory nerve endings and cell types in the cat's anteroventral cochlear nucleus seen with the Golgi method and Nomarski optics. J Comp Neurol 160:491-506.

Cant NB, Hyson RL (1992) Projections from the lateral nucleus of the trapezoid body to the medial superior olivary nucleus in the gerbil. Hear Res 58:26-34.

Carney LH (2002) A monaural cross-frequency coincidence-detection model for masked detection of low-frequency tones (Abstract 742). Presented at Midwinter Meeting of the Association for Research in Otolaryngology, St. Petersburg Beach, FL, January.

Caspary DM, Palombi SP, Backoff PM, Helfert RH, Finlayson PG (1993) GABA and glycine inputs control discharge rate within the excitatory response area of primary-like and phase-locked AVCN neurons. In: The mammalian cochlear nuclei: organization and function (Merchan MA, ed), pp 239-252. New York: Plenum.

Caspary DM, Backoff PM, Finlayson PG, Palombi PS (1994) Inhibitory inputs modulate discharge rate within frequency receptive fields of anteroventral cochlear nucleus neurons. J Neurophysiol 72:2124-2133.

Covey E, Jones DR, Casseday JH (1984) Projections from the superior olivary complex to the cochlear nucleus in the tree shrew. J Comp Neurol 226:289-305.

Ebert U, Ostwald J (1995a) GABA can improve acoustic contrast in the rat ventral cochlear nucleus. Exp Brain Res 104:310-322.

Ebert U, Ostwald J (1995b) GABA alters the discharge pattern of chopper neurons in the rat ventral cochlear nucleus. Hear Res 91:160-166.

Ferragamo MJ, Golding NL, Oertel D (1998) Synaptic inputs to stellate cells in the ventral cochlear nucleus. J Neurophysiol 79:51-63.

Galambos R, Davis H (1943) The response of single auditory nerve fibers to acoustic stimulation. J Neurophysiol 6:39-57.

Glaser EM, Ruchkin DS (1976) Principles of neurobiological signal analysis. New York: Academic.

Goldberg JM, Brownell WE (1973) Discharge characteristics of neurons in anteroventral and dorsal cochlear nuclei of cat. Brain Res 64:35-54.

Havey DC, Caspary DM (1980) A simple technique for constructing "piggy-back" multibarrel microelectrodes. Electroencephalogr Clin Neurophysiol 48:249-251.

Irvine DRF (1986) The auditory brainstem. In: Progress in sensory physiology. New York: Springer.

Kolston J, Osen KK, Hackney CM, Ottersen OP, Storm-Mathisen J (1992) An atlas of glycine- and GABA-like immunoreactivity and colocalization in the cochlear nuclear complex of the guinea pig. Anat Embryol (Berl) 186:443-465.

Kopp-Scheinpflug C (1999) Interaktion von Exzitation und Inhibition bei der zentralnervösen Verarbeitung akustischer Information. Aachen, Germany: Shaker Verlag.

Kopp-Scheinpflug C, Lippe WR, Dörrscheidt GJ, Rübsamen R (2002) The medial nucleus of the trapezoid body in the gerbil is more than a relay: comparison of pre- and postsynaptic activity. J Assoc Res Otolaryngol 8:8.
Manis PB, Marx SO (1991) Outward currents in isolated ventral cochlear nucleus neurons. J Neurosci 11:2865-2880.

Müller M (1996) The cochlear place-frequency map of the adult and developing Mongolian gerbil. Hear Res 94:148-156.

Müller M, Laube B, Burda H, Bruns V (1992) Structure and function of the cochlea in the African mole rat (Cryptomys hottentotus): evidence for a low frequency acoustic fovea. J Comp Physiol [A] 171:469-476.

Nelken I, Young ED (1994) Two separate inhibitory mechanisms shape the responses of dorsal cochlear nucleus type IV units to narrowband and wideband stimuli. J Neurophysiol 71:2446-2462.

Oertel D, Wickesberg RE (1993) Glycinergic inhibition in the cochlear nuclei: evidence for tuberculoventral neurons being glycinergic. In: The mammalian cochlear nuclei: organization and function (Merchan MA, ed), pp 225-237. New York: Plenum.

Oertel D, Wu SH, Garb MW, Dizack C (1990) Morphology and physiology of cells in slice preparations of the posteroventral cochlear nucleus of mice. J Comp Neurol 295:136-154.

Ohlemiller KK, Echteler SM (1990) Functional correlates of characteristic frequency in single cochlear nerve fibers of the Mongolian gerbil. J Comp Physiol [A] 167:329-338.

Ohlemiller KK, Echteler SM, Siegel JH (1991) Factors that influence rate-versus-intensity relations in single cochlear nerve fibers of the gerbil. J Acoust Soc Am 90:274-287.

Ostapoff EM, Feng JJ, Morest DK (1994) A physiological and structural study of neuron types in the cochlear nucleus. II. Neuron types and their structural correlation with response properties. J Comp Neurol 346:19-42.

Pfeiffer RR (1966a) Anteroventral cochlear nucleus: wave forms of extracellularly recorded spike potentials. Science 154:667-668.

Pfeiffer RR (1966b) Classification of response patterns of spike discharges for units in the cochlear nucleus: tone-burst stimulation. Exp Brain Res 1:220-235.

Rabiner LR, Gold B (1975) Theory and application of digital signal processing. Englewood Cliffs, NJ: Prentice-Hall.

Rhode WS, Greenberg S (1994) Lateral suppression and inhibition in the cochlear nucleus of the cat. J Neurophysiol 71:493-514.

Roberts RC, Ribak CE (1987) GABAergic neurons and axon terminals in the brainstem auditory nuclei of the gerbil. J Comp Neurol 258:267-280.

Rose JE, Kitzes LM, Gibson MM, Hind JE (1974) Observations on phase-sensitive neurons of anteroventral cochlear nucleus of the cat: nonlinearity of cochlear output. J Neurophysiol 37:218-253.

Rothman JS, Young ED, Manis PB (1993) Convergence of auditory nerve fibers onto bushy cells in the ventral cochlear nucleus: implications of a computational model. J Neurophysiol 70:2562-2583.

Rouiller EM, Cronin-Schreiber R, Fekete DM, Ryugo DK (1986) The central projections of intracellularly labeled auditory nerve fibers in cats: an analysis of terminal morphology. J Comp Neurol 249:261-278.

Rübsamen R, Gutowski M, Langkau J, Dörrscheidt GJ (1994) Growth of central nervous system auditory and visual nuclei in the postnatal gerbil (Meriones unguiculatus). J Comp Neurol 346:289-305.

Ryugo DK, Sento S (1991) Synaptic connections of the auditory nerve in cats: relationship between endbulbs of Held and spherical bushy cells. J Comp Neurol 305:35-48.

Sachs MB, Kiang NY (1968) Two-tone inhibition in auditory-nerve fibers. J Acoust Soc Am 43:1120-1128.

Schmiedt RA (1989) Spontaneous rates, thresholds and tuning of auditory-nerve fibers in the gerbil: comparisons to cat data. Hear Res 42:23-35.

Schofield BR (1991) Superior paraolivary nucleus in the pigmented guinea pig: separate classes of neurons project to the inferior colliculus and the cochlear nucleus. J Comp Neurol 312:68-76.

Schofield BR (1994) Projections to the cochlear nuclei from principal cells in the medial nucleus of the trapezoid body in guinea pigs. J Comp Neurol 344:83-100.

Shofner WP, Young ED (1985) Excitatory/inhibitory response types in the cochlear nucleus: relationships to discharge patterns and responses to electrical stimulation of the auditory nerve. J Neurophysiol 54:917-939.

Smith PH, Rhode WS (1989) Structural and functional properties distinguish two types of multipolar cells in the ventral cochlear nucleus. J Comp Neurol 282:595-616

Smith PH, Joris PX, Yin TC (1993) Projections of physiologically characterized spherical bushy cell axons from the cochlear nucleus of the cat: evidence for delay lines to the medial superior olive. J Comp Neurol 331:245-260.

Snyder RL, Leake PA (1988) Intrinsic connections within and between cochlear nucleus subdivisions in cat. J Comp Neurol 278:209-225.

Spirou GA, Brownell WE, Zidanic M (1990) Recordings from cat trapezoid body and HRP labeling of globular bushy cell axons. J Neurophysiol 63:1169-1190.

Walsh EJ, McGee J, Fitzakerley JL (1990) GABA actions within the caudal cochlear nucleus of developing kittens. J Neurophysiol 64:961-977. 
Warr WB, Beck JE (1996) Multiple projections from the ventral nucleus of the trapezoid body in the rat. Hear Res 93:83-101.

Wenthold RJ, Huie D, Altschuler RA, Reeks KA (1987) Glycine immunoreactivity localized in the cochlear nucleus and superior olivary complex. Neuroscience 22:897-912.

Wickesberg RE, Oertel D (1988) Tonotopic projection from the dorsal to the anteroventral cochlear nucleus of mice. J Comp Neurol 268:389-399.

Wickesberg RE, Oertel D (1990) Delayed, frequency-specific inhibition in the cochlear nuclei of mice: a mechanism for monaural echo suppression. J Neurosci 10:1762-1768.

Wiener MC, Richmond BJ (1999) Using response models to estimate channel capacity for neuronal classification of stationary visual stimuli using temporal coding. J Neurophysiol 82:2861-2875.
Winter IM, Palmer AR (1990) Responses of single units in the anteroventral cochlear nucleus of the guinea pig. Hear Res 44:161-178.

Winter IM, Robertson D, Yates GK (1990) Diversity of characteristic frequency rate-intensity functions in guinea pig auditory nerve fibres. Hear Res 45:191-202.

Wu SH, Oertel D (1986) Inhibitory circuitry in the ventral cochlear nucleus is probably mediated by glycine. J Neurosci 6:2691-2706.

Young ED, Voigt HF (1982) Response properties of type II and type III units in dorsal cochlear nucleus. Hear Res 6:153-169.

Young ED, Robert JM, Shofner WP (1988) Regularity and latency of units in ventral cochlear nucleus: implications for unit classification and generation of response properties. J Neurophysiol 60:1-29. 